\title{
Implicit 3-D Dyadic Green's Function Using Self-Adjoint Operators for Inhomogeneous Planar Ferrite Circulator With Vertically Layered External Material Employing Mode-Matching
}

\author{
Clifford M. Krowne, Senior Member, IEEE
}

\begin{abstract}
Self-adjoint operators are found for the differential equations describing the $z$-dependent field variation in the medium external to the ferrite microstrip circulator puck. The external medium is, in general, inhomogeneously layered, consisting of media with permittivity properties, magnetic properties, or both. Eigenvalue equations characterizing the radially sectioned medium outside the puck are found, as are the eigenvectors. When the $z$-dependent parts are multiplied with the radial and azimuthal dependences, the complete three-dimensional (3-D) field expressions are determined. Source-constraint equations (representing microstrip lines) driving the circulator are then combined with the mode-matching technique to obtain in direct space, implicit dyadic Green's function elements. Mode orthogonality is employed to encourage sparsity in matrix system development where appropriate or convenient. The implicit Green's function is particularly useful because field information and $s$-parameters may be found in real space, completely avoiding typical inverse transformations.
\end{abstract}

Index Terms-Dyadic Green's function, inhomogeneous ferrite, microstrip circulator, mode-matching, self-adjoint operator.

\section{INTRODUCTION}

D EVELOPING Green's-function approaches for canonical structures can be particularly advantageous when solving inhomogeneous boundary-valued problems, as is the case for planar circulator problems of the microstrip variety. The driving force occurs on the $r=R$ surface at the point $\phi=\phi^{\prime}$ and $z=z^{\prime}$ or on a strip at $\phi=\phi^{\prime}$. Obtaining explicit dyadic Green's-function expressions is known to be very convenient and allows extremely rapid numerical computation of electromagnetic fields and $s$-parameters [1], [2]. In those papers, the circulator was a circular ferrite puck, but with completely arbitrary radial variation of the descriptive parameters of the problem. The puck itself was made up of a number of annular rings, each with different widths, and with different material properties for the magnetization $M_{s}$ and demagnetization factor $N_{z z}$. The magnetic biasing field $H_{\text {app }}$ was also allowed to vary in an arbitrary radial manner.

Manuscript received May 1, 1997; revised January 14, 1998.

The author is with Code 6850.3, Microwave Technology Branch, Electronics Science \& Technology Division, Naval Research Laboratory, Washington, DC 20375-5347 USA.

Publisher Item Identifier S 0018-9480(98)02727-6.
Two- (2-D) and three-dimensional (3-D) dyadic Green's functions were obtained which depended upon recursive relations to find final expressions. Although these expressions are compact and explicit, the recursive nature of the development necessarily contains embedded information, making the actual algebraic dyadic Green's functions immensely complicated. Therefore, computer techniques are essential in studying the behavior of the dyadic Green's functions. But because of the canonical nature of the structure geometry, and the theoretical techniques employed in the derivations, these dyadic Green's functions lead to field evaluations between 1000-10000 times faster than intensive numerical techniques like finite-difference or finite-element methods.

What we are desirous of doing in this paper is dropping this complex inhomogeneous puck into a medium which consists of radial zones beyond the circulator puck perimeter. Each zone is made up of an arbitrary number of horizontal layers, stacked vertically in the $z$-direction. This arrangement outside of the puck will constitute yet another inhomogeneous problem, in addition to that of the puck itself. In principle, the region above the puck, bounded on the lower side by an electric wall formed by a microstrip conductor, and on the top by a metal limiting wall, can also be viewed as a zone.

Such a structure as outlined above can be used to treat the case where a circulator ferrite puck is dropped into a hole in a substrate, and possibly covered by a superstrate. Both the substrate and superstrate may be broken up into layers. All of the material external to the puck will be considered to be isotropic, but with the possibility that the cylinder above the puck and the layers in the radial zones can have permittivity properties, permeability properties (unbiased), or both simultaneous permittivity and permeability properties. Each radial zone, stretching vertically from the lower ground plane to the top horizontal wall, made up of many different layered regions, is viewed as a waveguide section, with a collective radial waveguide propagation constant. At a cylindrical wall $r=r_{j}$, mode-matching is applied. The $j$ index increases in value from $j=1$ at the puck-external medium interface $r_{1}=R$, to $j_{N}$ at the last interface. The last interface may be chosen as open, in which case a radiation condition could be applied, or as an electric or magnetic wall requiring explicit, but simple, 


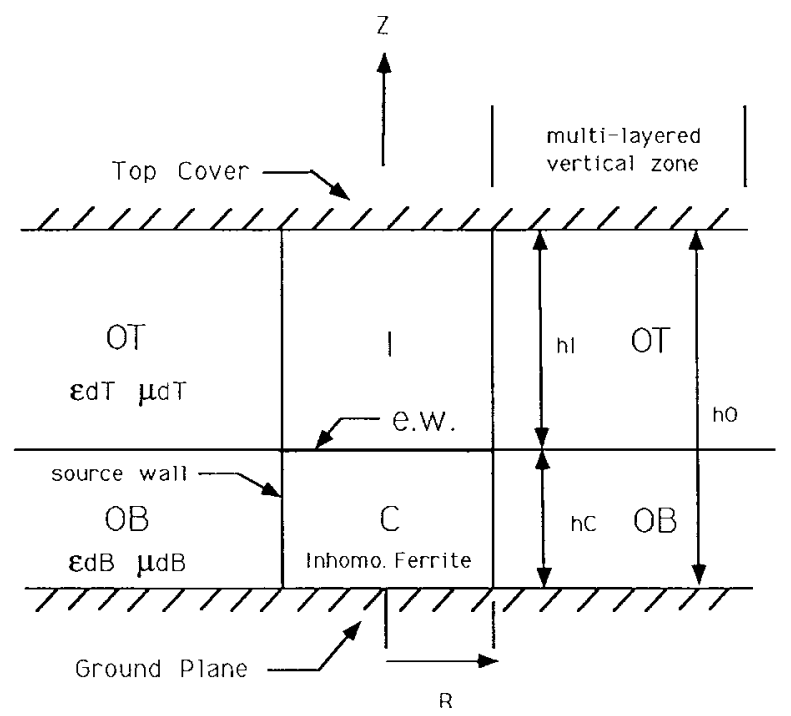

Fig. 1. The ferrite circulator structure including the regions above and surrounding the device. This figure is formed by taking a cut plane at $\phi=$ const (in 3-D).

mode-matching conditions for the last zone's vertically stacked regions.

Here we will treat a specific case of the general 3-D situation outlined in the last paragraph. The puck will be placed inside a substrate like that found in microwave monolithic integrated circuits (MMIC's), with a ground plane bounding it from below. An electric wall, representing microstrip metal, will constrain the fields within the ferrite puck material from above, and this electric wall will be flush with the substrate surface. Immediately above the puck will be isotropic material, not necessarily the same as that for the medium beyond the circulator puck perimeter. One zone exists beyond the perimeter, and it consists of the substrate on the bottom and another material region on top, not necessarily the same as the inner zone above the puck. The top layer, consisting of an inner and outer radial ordered set, constitutes the superstrate, which could be chosen by default in the simplest situation to be air.

For the substrate being part of a MMIC, it could be one of a number of semiconductor materials like $\mathrm{Si}$, GaAs, or even heterostructure material. For the case where a more hybrid-like circuit is used, it could be an unbiased magnetic material, even the same or related to that used for the puck itself. Furthermore, depending upon how the biasing magnetic field is obtained for the puck, the electric wall above the puck may be a microstrip-keeper metal combination to allow self-biasing of the ferrite material in the puck. With the use of a conventional biasing magnet, the origin of this field is considered to come from outside the whole structure, shown schematically in Fig. 1. Allowing for a magnet to be placed in a layered arrangement above the puck constitutes a greater complication to the problem which will not be addressed in this paper, although the theoretical principles for accomplishing such inclusion will be treated in this work.

Self-adjoint operators are found for the differential equations describing the $z$-dependent field variation in the medium zone external to the circulator puck. The external medium is, in general, inhomogeneously layered, consisting of media with permittivity properties, magnetic properties, or both. For the simplest case in which each zone has regions of only one trait (i.e., not mixed), and that trait is dielectric, information is available on the TE axisymmetric eigenvalue equation [3], eigenvector forms [4], [5], or scalar potential governing equations and the eigenvalues and eigenvectors using selfadjointness properties [6]. Eigenvalue equations characterizing the radially sectioned medium outside the puck are found, as are the eigenvectors. When the $z$-dependent parts are multiplied with the radial and azimuthal dependences, the complete field expressions are determined. Source-constraint equations driving the circulator are then combined with the modematching technique to obtain in direct space implicit dyadic Green's-function elements. Mode orthogonality is employed to encourage sparsity in matrix system development where appropriate or convenient. The self-adjoint operators lead to testing functions which may be used to test field continuity equations, thereby reducing some infinite summations to single-term contributions. The implicit Green's function is particularly useful because field information and $s$-paramaters may be found in real space, completely avoiding typical inverse transformations.

Consideration of field extension into the surrounding medium, beyond the circulator perimeter, including fringing such that fields may extend out and then above the height of the circulator nonreciprocal puck, is an essentially physical motivation for this theoretical work. The approach is a good approximation to a very complicated geometric and inhomogeneous problem, given the irregular effects arising from application of the dc-biasing magnetic field and the actual finite-width microstrip input and output lines. For narrow microstrip lines, the expectation that the fields extend beyond the device perimeter with azimuthal symmetry is very good, and essential to this canonical treatment. When some of the microstrip lines attain widths which are a noticeable fraction of the puck radius, the error introduced by the symmetry assumption for $r>R$ for the fields will be directly related to the fraction of the circumference occluded by the presence of the line itself.

\section{SELF-AdJOINT OPERATORS}

Consider the situation where the electromagnetic field occupies three areas (see Fig. 1). The first area $\mathrm{C}$ is that filled by the ferrite nonreciprocal material for $r<R$ and $0 \leq z \leq h_{\mathrm{C}}$. The second area or zone $\mathrm{O}$ has two regions for $r>R$, a bottom region $\mathrm{OB}$ with $0 \leq z \leq h_{\mathrm{C}}$ and a top region $\mathrm{OT}$ with $h_{\mathrm{C}} \leq z \leq h_{\mathrm{O}}$. And the third region $\mathrm{I}$ has $r<R$ and $h_{\mathrm{C}} \leq z \leq h_{\mathrm{O}}$. Conducting walls are assumed at $z=0, h_{\mathrm{O}}$ (all $r)$ and $z=h_{\mathrm{C}}(r<R)$, and the radiation condition in effect as $r \rightarrow \infty$. Fig. 2 shows a cross section through a $z=$ const plane, with microstrip lines coming into (or out of) the ports located at $\phi_{1}, \phi_{2}, \cdots, \phi_{i}, \cdots, \phi_{N_{p}}$ on the $r=R$ puck surface.

In order to maintain the same field structure formulas and parallel construction techniques inside and outside of the puck, governing equations are developed in the zone $r>R$ with the 


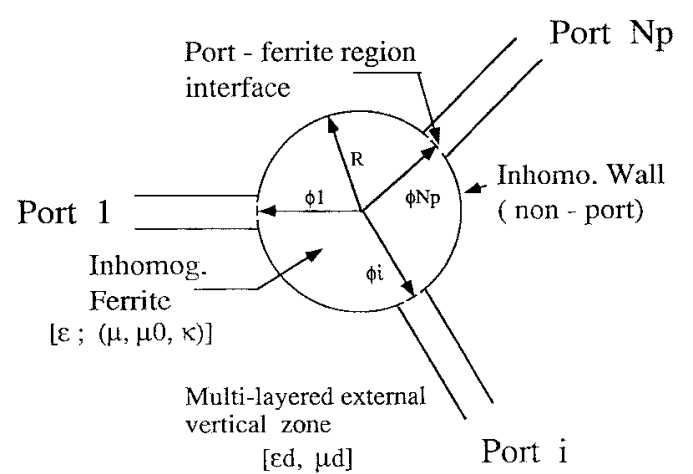

Fig. 2. This sketch represents a cut plane at $z=$ const (in 3-D) for the ferrite circulator structure.

stacked OB and OT regions utilizing field components from the puck in the isotropic limit. Specifically, $\mathrm{TM}_{z}$ (e-mode) and $\mathrm{TE}_{z}$ ( $h$-mode) equations are sought. Maxwell's sourceless equations assuming $e^{i \omega t}$ dependence are

$$
\begin{aligned}
& \nabla \times \boldsymbol{E}=-i \omega \mu \boldsymbol{H} \\
& \nabla \times \boldsymbol{H}=i \omega \varepsilon \boldsymbol{E} .
\end{aligned}
$$

\section{A. $\mathrm{TM}_{z}$ Operator Properties}

Take the curl of (1a), noting that both $\mu$ and $\varepsilon$ depend upon spatial location, namely that $\mu=\mu(\boldsymbol{r})=\mu(r, \phi, z)$ and $\varepsilon=\varepsilon(\boldsymbol{r})=\varepsilon(r, \phi, z)$

$$
\begin{aligned}
\nabla \times \nabla \times \boldsymbol{E} & =-i \omega \nabla \times(\mu \boldsymbol{H}) \\
& =\omega^{2} \mu \varepsilon \boldsymbol{E}+\frac{\nabla \mu(\boldsymbol{r})}{\mu} \times \nabla \times \boldsymbol{E}
\end{aligned}
$$

using both curl expressions (1) to remove any $H$-field dependence. Using an identity to eliminate the curlcurl term

$$
\nabla^{2} \boldsymbol{E}-\nabla(\nabla \cdot \boldsymbol{E})+\omega^{2} \mu \varepsilon \boldsymbol{E}+\frac{\nabla \mu(r)}{\mu} \times \nabla \times \boldsymbol{E}=0
$$

Realizing that the divergence of a curl in the left-hand side (LHS) of (1b) is zero, the divergence of the electric field in (3) may be replaced by

$$
\begin{aligned}
\nabla \cdot \boldsymbol{E} & =-\frac{\nabla \varepsilon(\boldsymbol{r})}{\varepsilon} \cdot \boldsymbol{E} \\
& =-\frac{1}{\varepsilon} \frac{d \varepsilon(z)}{d z} \hat{z} \cdot \boldsymbol{E} \\
& =-\frac{1}{\varepsilon} \frac{d \varepsilon(z)}{d z} E_{z} .
\end{aligned}
$$

Equation (4) has been obtained from the general spatial variation being reduced to that in only the $z$-direction because of regional changes within a zone (i.e., for a two-region zone as being considered here, $\varepsilon(z)=\varepsilon_{d}^{\mathrm{OB}}$ for $0 \leq z \leq h_{\mathrm{C}}$ and $\varepsilon(z)=\varepsilon_{d}^{\mathrm{OT}}$ for $h_{\mathrm{C}} \leq z \leq h_{\mathrm{O}}$ with a discontinuity at $z=h_{\mathrm{C}}$ ).

Inserting (4) into (3), the vector electric-field governing equation is found:

$$
\begin{gathered}
\nabla^{2} \boldsymbol{E}+\nabla\left(\frac{1}{\varepsilon} \frac{d \varepsilon(z)}{d z} E_{z}\right)+k^{2} \boldsymbol{E}+\frac{1}{\mu} \frac{d \mu(z)}{d z} \\
\cdot\left\{\left(\frac{1}{r} \frac{\partial E_{z}}{\partial \phi}-\frac{\partial E_{\phi}}{\partial z}\right) \hat{\phi}-\left(\frac{1}{r} \frac{\partial E_{r}}{\partial z}-\frac{\partial E_{z}}{\partial r}\right) \hat{r}\right\}=0 \\
k^{2}=\omega^{2} \mu \varepsilon=\omega^{2} \mu(\boldsymbol{r}) \varepsilon(\boldsymbol{r})=\omega^{2} \mu(z) \varepsilon(z) .
\end{gathered}
$$

If the TM to $z$ is selected as the basic mode electric type (out of the two required for a complete field description) with only the electric field existing perpendicular to the transverse plane, this being precisely the same directional field setup inside the puck, then (5) vastly simplifies to

$$
\begin{aligned}
& \nabla^{2} E_{z}+\frac{\partial}{\partial z}\left(\frac{1}{\varepsilon} \frac{d \varepsilon(z)}{d z} E_{z}\right)+k^{2} E_{z}=0 \\
& \frac{1}{r} \frac{\partial}{\partial r}\left(r \frac{\partial E_{z}}{\partial r}\right)+\frac{1}{r^{2}} \frac{\partial^{2} E_{z}}{\partial \phi^{2}}+\frac{\partial^{2} E_{z}}{\partial z^{2}}+\frac{\partial}{\partial z}\left(\frac{1}{\varepsilon} \frac{d \varepsilon(z)}{d z} E_{z}\right) \\
& \quad+k^{2} E_{z}=0 .
\end{aligned}
$$

Since all of the other field components (transverse) depend on formulas written in terms of $E_{z}$, only its expansion need be considered first:

$$
\begin{aligned}
E_{z}^{e} & (r, \phi, z) \\
& =\sum_{n=-\infty}^{\infty} \sum_{k_{z}=f}^{b} R_{n k_{z}}^{e}(r) e^{i k_{z} z} A_{n k_{z}}^{e \mathrm{O}} e^{i n \phi} \\
& =\sum_{n=-\infty}^{\infty} \sum_{k_{z}=b} R_{n k_{z}}^{e}(r)\left[e^{i k_{z} z} A_{n k_{z}}^{e \mathrm{O}}+e^{-i k_{z} z} A_{n,-k_{z}}^{e \mathrm{O}}\right] e^{i n \phi} \\
& =\sum_{n=-\infty}^{\infty} \sum_{k_{z}=b} R_{n k_{z}}^{e}(r)\left[e^{i k_{z} z} A_{n k_{z}}^{e \mathrm{O}-}+e^{-i k_{z} z} A_{n k_{z}}^{e \mathrm{O}+}\right] e^{i n \phi} \\
& =\sum_{n=-\infty}^{\infty} \sum_{k_{z}=b} R_{n k_{z}}^{e}(r) Z_{k_{z}}^{e}(z) e^{i n \phi} \\
& =\sum_{n=-\infty}^{\infty} \sum_{\sigma} R_{n \sigma}^{e}(r) Z_{\sigma}^{e}(z) e^{i n \phi} \\
& =\sum_{n=-\infty}^{\infty} \sum_{m_{e}=1}^{\infty} R_{n m_{e}}^{e}(r) Z_{m_{e}}^{e}(z) e^{i n \phi} .
\end{aligned}
$$

Here it is seen that the electric-field solution is the sum over the radial variation, $z$-directed harmonic, and azimuthal harmonic products. The $A$ coefficient provides the term by term weighting. The infinite sum over $k_{z}$ propagation constants in forward $(f)$ and backward $(b)$ directions may be changed into only a backward wave summation, but now requiring explicit forward and backward $A$ coefficients. Superscripts "-" and "+" added to the $A$ coefficients store that wavedirection information, letting the subscript propagation index being simplified to merely $k_{z}=b$. This process is collected together in the next line. Recognizing that in each zone only the radial propagation constant $\sigma$ is definable (and, therefore, capable of being indexed over the entire zone), but that $k_{z}$ varies from region to region within a zone, the fifth line is obtained. Finally, the $\sigma$ solutions to be determined later can be assigned for the solutions, index numbers ordered as $m_{e}=1,2, \cdots, \infty$. The associations for $Z_{m_{e}}^{e}(z)$ in (9) can be summarized as

$$
\begin{aligned}
Z_{m_{e}}^{e}(z) & =Z_{\sigma}^{e}(z) \\
& =Z_{k_{z}}^{e}(z) \\
& =e^{i k_{z} z} A_{n k_{z}}^{e \mathrm{O}-}+e^{-i k_{z} z} A_{n k_{z}}^{e \mathrm{O}} \\
& =e^{i k_{z} z} A_{n k_{z}}^{e \mathrm{O}}+e^{-i k_{z} z} A_{n,-k_{z}}^{e \mathrm{O}} .
\end{aligned}
$$


Now, inserting (9) into (8) and applying separation of variables, with

$$
\lambda_{m_{e}}^{e}=-\left(\sigma_{m_{e}}^{\mathrm{O}}\right)^{2}
$$

acting as the separation constant

$$
\begin{array}{r}
\frac{1}{r} \frac{d}{d r}\left(r R_{m_{e}}^{e}(r)\right)-\left(\lambda_{m_{e}}^{e}+\frac{n^{2}}{r^{2}}\right) R_{m_{e}}^{e}(r)=0 \\
\frac{d^{2} Z_{m_{e}}^{e}(z)}{d z^{2}}+\frac{d}{d z}\left\{\frac{1}{\varepsilon} \frac{d \varepsilon}{d z} Z_{m_{e}}^{e}(z)\right\}+\left(k^{2}+\lambda_{m_{e}}^{e}\right) Z_{m_{e}}^{e}(z)=0 .
\end{array}
$$

Select the first two derivative terms of (13), plus the first of the last bracketed sum, as the inhomogeneous linear operator $L_{\mathrm{TM}}$ :

$$
L_{\mathrm{TM}}=\frac{d^{2}}{d z^{2}}+\frac{d}{d z}\left\{\frac{1}{\varepsilon} \frac{d \varepsilon}{d z}[]\right\}+k^{2}
$$

Invoking (14) enables (13) to be recast as

$$
L_{\mathrm{TM}} Z_{m_{e}}^{e}(z)=-\lambda_{m_{e}}^{e} Z_{m_{e}}^{e}(z) .
$$

Operator equation (15) is in eigenvalue form, the eigenvalue operator on the right-hand side (RHS) of the equation merely the eigenvalue constant. Requiring the radiation condition to hold as $r \rightarrow \infty$ makes

$$
R_{m_{e}}^{e}(r)=K_{n}\left(\sigma_{m_{e}}^{\mathrm{O}} r\right)
$$

Next, let us find the adjoint of $L$ to better understand the behavior of the inhomogeneously loaded waveguide zone. For ease of mathematical argument and brevity, abbreviate two different $Z_{m_{e}}^{e}(z)$ solutions as $u$ and $v$ and define the inner product on the interval $\left(0, h_{\mathrm{O}}\right)$ as

$$
\langle v, u\rangle=\int_{0}^{h_{\mathrm{O}}} w(z) v u d z
$$

with weight $w(z)$. To find the adjoint, study

$$
\langle v, L u\rangle=\int_{0}^{h_{\mathrm{O}}} w(z) v\{L u\} d z .
$$

Sometimes it is convenient to explicitly place the weight in the bracketed expression when we wish to be reminded of its presence, as in $\langle w v, L u\rangle$. Anyway, we seek to convert (18) into the form $\left\langle L^{a} v, u\right\rangle$ by repeated application of integration by parts and thereby identify the adjoint form $L^{a}$ of the operator $L$. The weight we choose here is

$$
w(z)=\varepsilon(z) .
$$

Therefore,

$$
\begin{aligned}
\langle v & \left.L_{\mathrm{TM}} u\right\rangle \\
& =\int_{0}^{h_{\mathrm{O}}} v\left[\frac{d^{2} u}{d z^{2}}+\frac{d}{d z}\left\{\frac{1}{\varepsilon} \frac{d \varepsilon}{d z} u\right\}+k^{2} u\right] \varepsilon(z) d z \\
& =\int_{0}^{h_{\mathrm{O}}} v\left[\frac{1}{\varepsilon} \varepsilon \frac{d^{2} u}{d z^{2}}+\frac{1}{\varepsilon} \frac{d \varepsilon}{d z} \frac{d u}{d z}+u \frac{d}{d z}\left(\frac{1}{\varepsilon} \frac{d \varepsilon}{d z}\right)+k^{2} u\right] \varepsilon(z) d z \\
& =\int_{0}^{h_{\mathrm{O}}} v\left[\frac{1}{\varepsilon} \frac{d}{d z}\left(\varepsilon \frac{d u}{d z}\right)+u \frac{d}{d z}\left(\frac{1}{\varepsilon} \frac{d \varepsilon}{d z}\right)+k^{2} u\right] \varepsilon(z) d z
\end{aligned}
$$

$$
\begin{aligned}
& =\int_{0}^{h_{0}} v \frac{1}{\varepsilon} \frac{d}{d z}\left(\varepsilon \frac{d u}{d z}\right) \varepsilon(z) d z \\
& +\int_{0}^{h_{\mathrm{O}}} v\left[u \frac{d}{d z}\left(\frac{1}{\varepsilon} \frac{d \varepsilon}{d z}\right)+k^{2} u\right] \varepsilon(z) d z \\
& =\int_{0}^{h_{0}} v \frac{1}{\varepsilon} \frac{d}{d z}\left(\varepsilon \frac{d u}{d z}\right) \varepsilon(z) d z+\int_{0}^{h_{\circ}} v q(z) u \varepsilon(z) d z \\
& =\int_{0}^{h_{0}} v \frac{d}{d z}\left(\varepsilon \frac{d u}{d z}\right) d z+\langle q v, u\rangle \\
& =\int_{0}^{h_{\mathrm{O}}} v d\left(\varepsilon \frac{d u}{d z}\right)+\langle q v, u\rangle \\
& =\int_{0}^{h_{\circ}}\left\{d\left[v \varepsilon \frac{d u}{d z}\right]-\varepsilon \frac{d u}{d z} d v\right\}+\langle q v, u\rangle \\
& =\left.v \varepsilon \frac{d u}{d z}\right|_{0} ^{h_{\circ}}-\int_{0}^{h_{\circ}} \varepsilon \frac{d u}{d z} d v+\langle q v, u\rangle \\
& =v\left(h_{\mathrm{O}}\right) \varepsilon\left(h_{\mathrm{O}}\right) \frac{d u}{d z}\left(h_{\mathrm{O}}\right)-v(0) \varepsilon(0) \frac{d u}{d z}(0) \\
& -\int_{0}^{h_{\circ}} \varepsilon \frac{d u}{d z} d v+\langle q v, u\rangle \\
& =-\int_{0}^{h_{\odot}} \varepsilon \frac{d u}{d z} d v+\langle q v, u\rangle \\
& =-\int_{0}^{h_{\odot}} \varepsilon \frac{d u}{d z} \frac{d v}{d z} d v+\langle q v, u\rangle \\
& =-\int_{0}^{h_{O}} \varepsilon \frac{d v}{d z} d u+\langle q v, u\rangle \\
& =-\int_{0}^{h_{\circ}}\left\{d\left[u \varepsilon \frac{d v}{d z}\right]-u d\left[\varepsilon \frac{d v}{d z}\right]\right\}+\langle q v, u\rangle \\
& =-\left.u \varepsilon \frac{d v}{d z}\right|_{0} ^{h_{\odot}}+\int_{0}^{h_{\odot}} u d\left[\varepsilon \frac{d v}{d z}\right]+\langle q v, u\rangle \\
& =-u\left(h_{\mathrm{O}}\right) \varepsilon\left(h_{\mathrm{O}}\right) \frac{d v}{d z}\left(h_{\mathrm{O}}\right)+u(0) \varepsilon(0) \frac{d v}{d z}(0) \\
& +\int_{0}^{h_{\mathrm{O}}} u \frac{d}{d z}\left(\varepsilon \frac{d v}{d z}\right)+\langle q v, u\rangle \\
& =\int_{0}^{h_{\circ}} u \frac{d}{d z}\left(\varepsilon \frac{d v}{d z}\right)+\langle q v, u\rangle \\
& =\int_{0}^{h_{\circ}} u\left[\frac{1}{\varepsilon} \frac{d}{d z}\left(\varepsilon \frac{d v}{d z}\right)+v \frac{d}{d z}\left(\frac{1}{\varepsilon} \frac{d \varepsilon}{d z}\right)+k^{2} v\right] \quad \varepsilon(z) d z \\
& =\int_{0}^{h_{\circ}}\left[\frac{d^{2} v}{d z^{2}}+\frac{d}{d z}\left\{\frac{1}{\varepsilon} \frac{d \varepsilon}{d z} v\right\}+k^{2} v\right] u \varepsilon(z) d z \\
& =\left\langle L_{\mathrm{TM}}^{a} v, u\right\rangle \text {. }
\end{aligned}
$$

From (20), the adjoint form of the operator can be identified as

$$
L_{\mathrm{TM}}^{a}=L_{\mathrm{TM}} .
$$

Thus, it is seen that the operator is self-adjoint [7]-[9]. Equation (21) was obtained by using a number of boundary conditions, which will be briefly covered in this section. Electric-wall conditions at $z=0, h_{\mathrm{O}}$ require transversefield components $E_{r}$ and $E_{\phi}$ to be zero. From the $E_{\phi}$-field component expression [10], e.g.,

$$
E_{\phi j}^{\mathrm{O}}=-s_{j} \frac{\partial H_{z j}^{\mathrm{O}}}{\partial r}+\frac{p_{j}}{r} \frac{\partial E_{z j}^{\mathrm{O}}}{\partial \phi}
$$


one observes that pure Neumann conditions hold at the ends of the inner product domain. This conclusion follows because (22) originates when exponential (harmonic, plane waves) are chosen for the $z$-functional behavior. Thus, the coefficient $p_{j}$, from the second piece of $E_{\phi}$ generating the TM mode, indexed for $j z$-directed modes, contains an $i k_{z}$ factor, implying the presence of a partial derivative $\partial / \partial z$ operator.

$$
\begin{gathered}
\frac{d v}{d z}(0)=\left.\frac{d v}{d z}\right|_{z=0}=0 \\
\frac{d v}{d z}\left(h_{\mathrm{O}}\right)=\left.\frac{d v}{d z}\right|_{z=h_{\mathrm{O}}}=0 .
\end{gathered}
$$

The same conditions, of course, hold for $u$. Because within a region of a zone, $\varepsilon(z)$ is stipulated to vary continuously, and for the zone to vary, at most, piecewise continuously, because the discontinuities only occur at the region interfaces, $d \varepsilon / d z$ will be well behaved at the domain ends. Furthermore, requiring $\varepsilon(z)$ to be constant within a region makes a Neumann condition also hold for it. Thus

$$
\begin{gathered}
\frac{d \varepsilon}{d z}(0)=\left.\frac{d \varepsilon}{d z}\right|_{z=0}=0 \\
\frac{d \varepsilon}{d z}\left(h_{\mathrm{O}}\right)=\left.\frac{d \varepsilon}{d z}\right|_{z=h_{\mathrm{O}}}=0 .
\end{gathered}
$$

Neumann conditions (24) on $d \varepsilon(z) / d z$ are not required for obtaining the self-adjoint relation (20). This is also the case for TE modes, as will be demonstrated later.

It is emphasized here that although $L_{\mathrm{TM}}$ is self-adjoint, it is not representing lossless media. The media can be dielectrically lossy, magnetically lossy, or lossy in both regards simultaneously. Thus, the eigenvalue in (15) may be complex. In fact, for ordinary media, we expect it to be complex. Now, let us review in light of this fact, the short derivation of orthogonality as implied by self-adjointness. Consider that any self-adjoint operator $L$ obeys

$$
\langle L u, v\rangle=\langle u, L v\rangle .
$$

Let

$$
\begin{aligned}
& L_{\mathrm{TM}} u=-\lambda_{m_{e}}^{e} u \\
& L_{\mathrm{TM}} v=-\lambda_{m_{e}^{\prime}}^{e} v
\end{aligned}
$$

associating $u$ and $v$ with, respectively, the eigenvalue indices $m_{e}$ and $m_{e}^{\prime}$. Placing (26) into (25) yields

$$
\left\langle-\lambda_{m_{e}}^{e} u, v\right\rangle=\left\langle u,-\lambda_{m_{e}^{\prime}}^{e} v\right\rangle
$$

or

$$
\left(\lambda_{m_{e}^{\prime}}^{e}-\lambda_{m_{e}}^{e}\right)\langle u, v\rangle=0
$$

or

$$
\langle u, v\rangle=0
$$

Orthogonality relation (27) holds precisely because the $z$ eigenfunctions are associated with different eigenvalues. In our case, the eigenvalue difference is between different complex eigenvalues. Relation (27) says that the $z$-eigenfunctions are orthogonal no matter how many different regions are stacked in a zone, and this is true regardless of whether we use only dielectric regions, magnetic regions (unbiased), or intermix these two types of regions, or even if we further complicate the situation by using regions with both dielectric and magnetic characteristics.

\section{B. $\mathrm{TE}_{z}$ Operator Properties}

Following the philosophy of the $\mathrm{TM}_{z}$ development in Section II-A, take the curl of (1b):

$$
\begin{aligned}
\nabla \times \nabla \times \boldsymbol{H} & =i \omega \nabla \times(\varepsilon \boldsymbol{E}) \\
& =\omega^{2} \mu \varepsilon \boldsymbol{H}+\frac{\nabla \varepsilon(\boldsymbol{r})}{\varepsilon} \times \nabla \times \boldsymbol{H}
\end{aligned}
$$

using both curl expressions (1) to remove any $\boldsymbol{E}$-field dependence. Using an identity to eliminate the curlcurl term

$$
\nabla^{2} \boldsymbol{H}-\nabla(\nabla \cdot \boldsymbol{H})+\omega^{2} \mu \varepsilon \boldsymbol{H}+\frac{\nabla \varepsilon(r)}{\varepsilon} \times \nabla \times \boldsymbol{H}=0 .
$$

Realizing that the divergence of a curl in the LHS of (1a) is zero, the divergence of the magnetic field in (29) may be replaced by

$$
\begin{aligned}
\nabla \cdot \boldsymbol{H} & =-\frac{\nabla \mu(\boldsymbol{r})}{\mu} \cdot \boldsymbol{H} \\
& =-\frac{1}{\mu} \frac{d \mu(z)}{d z} \hat{z} \cdot \boldsymbol{H} \\
& =-\frac{1}{\mu} \frac{d \mu(z)}{d z} H_{z} \cdot
\end{aligned}
$$

Equation (30) has been obtained from the general spatial variation being reduced to that in only the $z$-direction because of regional changes within a zone (i.e., for a two-region zone as being considered here, $\mu(z)=\mu_{d}^{\mathrm{OB}}$ for $0 \leq z \leq h_{\mathrm{C}}$ and $\mu(z)=\mu_{d}^{\mathrm{OT}}$ for $h_{\mathrm{C}} \leq z \leq h_{\mathrm{O}}$ with a discontinuity at $z=h_{\mathrm{C}}$ ).

Inserting (30) into (29), the vector magnetic-field governing equation is found:

$$
\begin{aligned}
\nabla^{2} \boldsymbol{H} & +\nabla\left(\frac{1}{\mu} \frac{d \mu(z)}{d z} H_{z}\right)+k^{2} \boldsymbol{H}+\frac{1}{\varepsilon} \frac{d \varepsilon(z)}{d z} \\
\cdot & \left\{\left(\frac{1}{r} \frac{\partial H_{z}}{\partial \phi}-\frac{\partial H_{\phi}}{\partial z}\right) \hat{\phi}-\left(\frac{1}{r} \frac{\partial H_{r}}{\partial z}-\frac{\partial H_{z}}{\partial r}\right) \hat{r}\right\}=0 .
\end{aligned}
$$

If the TE to $z$ is selected as the basic mode magnetic type with only the magnetic field existing perpendicular to the transverse plane, then (31) greatly simplifies to

$$
\nabla^{2} H_{z}+\frac{\partial}{\partial z}\left(\frac{1}{\mu} \frac{d \mu(z)}{d z} H_{z}\right)+k^{2} H_{z}=0
$$

or

$$
\begin{aligned}
& \frac{1}{r} \frac{\partial}{\partial r}\left(r \frac{\partial H_{z}}{\partial r}\right)+\frac{1}{r^{2}} \frac{\partial^{2} H_{z}}{\partial \phi^{2}}+\frac{\partial^{2} H_{z}}{\partial z^{2}}+\frac{\partial}{\partial z}\left(\frac{1}{\mu} \frac{d \mu(z)}{d z} H_{z}\right) \\
& \quad+k^{2} H_{z}=0 .
\end{aligned}
$$

Since all of the other field components (transverse) depend on formulas written in terms of $H_{z}$, only its expansion need be 
found:

$$
\begin{aligned}
H_{z}^{h} & (r, \phi, z) \\
& =\sum_{n=-\infty}^{\infty} \sum_{k_{z}=f}^{b} R_{n k_{z}}^{h}(r) e^{i k_{z} z} A_{n k_{z}}^{h \mathrm{O}} e^{i n \phi} \\
& =\sum_{n=-\infty}^{\infty} \sum_{k_{z}=b} R_{n k_{z}}^{h}(r)\left[e^{i k_{z} z} A_{n k_{z}}^{h \mathrm{O}}+e^{-i k_{z} z} A_{n,-k_{z}}^{h \mathrm{O}}\right] e^{i n \phi} \\
& =\sum_{n=-\infty}^{\infty} \sum_{k_{z}=b} R_{n k_{z}}^{h}(r)\left[e^{i k_{z} z} A_{n k_{z}}^{h \mathrm{O}-}+e^{-i k_{z} z} A_{n k_{z}}^{h \mathrm{O}+}\right] e^{i n \phi} \\
& =\sum_{n=-\infty}^{\infty} \sum_{k_{z}=b} R_{n k_{z}}^{h}(r) Z_{k_{z}}^{h}(z) e^{i n \phi} \\
& =\sum_{n=-\infty}^{\infty} \sum_{\sigma} R_{n \sigma}^{h}(r) Z_{\sigma}^{h}(z) e^{i n \phi} \\
& =\sum_{n=-\infty}^{\infty} \sum_{m_{h}=1}^{\infty} R_{n m_{h}}^{h}(r) Z_{m_{h}}^{h}(z) e^{i n \phi} .
\end{aligned}
$$

Here it is seen that the magnetic-field solution is the sum over the radial variation, $z$-directed harmonic, and azimuthal harmonic products. The $\sigma$ solutions to be determined later, more correctly denoted as $\sigma_{m_{h}}$, are assigned for the solutions index numbers ordered as $m_{h}=1,2, \cdots, \infty$. Solutions $\sigma_{m_{h}}$ are different than $\sigma_{m_{e}}$ for the $\mathrm{TM}_{z}$ case. The associations for $Z_{m_{h}}^{h}(z)$ in (34) can be summarized as

$$
\begin{aligned}
Z_{m_{h}}^{h}(z) & =Z_{\sigma}^{h}(z) \\
& =Z_{k_{z}}^{h}(z) \\
& =e^{i k_{z} z} A_{n k_{z}}^{h \mathrm{O}-}+e^{-i k_{z} z} A_{n k_{z}}^{h \mathrm{O}+} \\
& =e^{i k_{z} z} A_{n k_{z}}^{h \mathrm{O}}+e^{-i k_{z} z} A_{n,-k_{z}}^{h \mathrm{O}} .
\end{aligned}
$$

Now inserting (34) into (33) and applying separation of variables with

$$
\lambda_{m_{h}}^{h}=-\left(\sigma_{m_{h}}^{\mathrm{O}}\right)^{2}
$$

acting as the separation constant

$$
\begin{array}{r}
\frac{1}{r} \frac{d}{d r}\left(r R_{m_{h}}^{h}(r)\right)-\left(\lambda_{m_{h}}^{h}+\frac{n^{2}}{r^{2}}\right) R_{m_{h}}^{h}(r)=0 \\
\frac{d^{2} Z_{m_{h}}^{h}(z)}{d z^{2}}+\frac{d}{d z}\left\{\frac{1}{\mu} \frac{d \mu}{d z} Z_{m_{h}}^{h}(z)\right\}+\left(k^{2}+\lambda_{m_{h}}^{h}\right) Z_{m_{h}}^{h}(z)=0 .
\end{array}
$$

Select the first two derivative terms of (38), plus the first of the last bracketed sum, as the inhomogeneous linear operator $L_{\mathrm{TE}}$ :

$$
L_{\mathrm{TE}}=\frac{d^{2}}{d z^{2}}+\frac{d}{d z}\left\{\frac{1}{\mu} \frac{d \mu}{d z}[]\right\}+k^{2} .
$$

Utilizing (39) enables (38) to be recast as

$$
L_{\mathrm{TE}} Z_{m_{h}}^{h}(z)=-\lambda_{m_{h}}^{h} Z_{m_{h}}^{h}(z) .
$$

Requiring that the radiation condition to hold again as $r \rightarrow \infty$ makes

$$
R_{m_{h}}^{h}(r)=K_{n}\left(\sigma_{m_{h}}^{\mathrm{O}} r\right) .
$$

Next, let us find the adjoint of $L$ to better understand the behavior of the inhomogeneously loaded waveguide zone. For ease of mathematical argument and brevity, again abbreviate two different $Z_{m_{h_{t}}}^{h}(z)$ solutions as $u$ and $v$ and use the inner product definitions (17) and (18) on the interval $\left(0, h_{\mathrm{O}}\right)$. We seek to convert (18) into the form $\left\langle L^{a} v, u\right\rangle$ in order to identify the adjoint form $L^{a}$ of the operator $L=L_{\mathrm{TE}}$. The weight we choose here is

$$
w(z)=\mu(z)
$$

Therefore,

$$
\begin{aligned}
& \left\langle v, L_{\mathrm{TE}} u\right\rangle \\
& =\int_{0}^{h_{\circ}} v\left[\frac{d^{2} u}{d z^{2}}+\frac{d}{d z}\left\{\frac{1}{\mu} \frac{d \mu}{d z} u\right\}+k^{2} u\right] \mu(z) d z \\
& =\int_{0}^{h_{\circ}} v\left[\frac{1}{\mu} \mu \frac{d^{2} u}{d z^{2}}+\frac{1}{\mu} \frac{d \mu}{d z} \frac{d u}{d z}+u \frac{d}{d z}\left(\frac{1}{\mu} \frac{d \mu}{d z}\right)\right. \\
& \left.+k^{2} u\right] \mu(z) d z \\
& =\int_{0}^{h_{\odot}} v\left[\frac{1}{\mu} \frac{d}{d z}\left(\mu \frac{d u}{d z}\right)+u \frac{d}{d z}\left(\frac{1}{\mu} \frac{d \mu}{d z}\right)+k^{2} u\right] \mu(z) d z \\
& =\int_{0}^{h_{\mathrm{O}}} v \frac{1}{\mu} \frac{d}{d z}\left(\mu \frac{d u}{d z}\right) \mu(z) d z \\
& +\int_{0}^{h_{\circ}} v\left[u \frac{d}{d z}\left(\frac{1}{\mu} \frac{d \mu}{d z}\right)+k^{2} u\right] \mu(z) d z \\
& =\int_{0}^{h_{\circ}} v \frac{1}{\mu} \frac{d}{d z}\left(\mu \frac{d u}{d z}\right) \mu(z) d z+\int_{0}^{h_{\odot}} v q(z) u \mu(z) d z \\
& =\int_{0}^{h_{\mathrm{O}}} v \frac{d}{d z}\left(\mu \frac{d u}{d z}\right) d z+\langle q v, u\rangle \\
& =\int_{0}^{h_{O}} v d\left(\mu \frac{d u}{d z}\right)+\langle q v, u\rangle \\
& =\int_{0}^{h_{\circ}}\left\{d\left[v \mu \frac{d u}{d z}\right]-\mu \frac{d u}{d z} d v\right\}+\langle q v, u\rangle \\
& =\left.v \mu \frac{d u}{d z}\right|_{0} ^{h_{\mathrm{O}}}-\int_{0}^{h_{\mathrm{O}}} \mu \frac{d u}{d z} d v+\langle q v, u\rangle \\
& =v\left(h_{\mathrm{O}}\right) \mu\left(h_{\mathrm{O}}\right) \frac{d u}{d z}\left(h_{\mathrm{O}}\right)-v(0) \mu(0) \frac{d u}{d z}(0) \\
& -\int_{0}^{h_{\odot}} \mu \frac{d u}{d z} d v+\langle q v, u\rangle \\
& =-\int_{0}^{h_{\mathrm{O}}} \mu \frac{d u}{d z} d v+\langle q v, u\rangle \\
& =-\int_{0}^{h_{O}} \mu \frac{d u}{d z} \frac{d v}{d z} d z+\langle q v, u\rangle \\
& =-\int_{0}^{h_{\circ}} \mu \frac{d v}{d z} d u+\langle q v, u\rangle \\
& =-\int_{0}^{h_{\odot}}\left\{d\left[u \mu \frac{d v}{d z}\right]-u d\left[\mu \frac{d v}{d z}\right]\right\}+\langle q v, u\rangle
\end{aligned}
$$




$$
\begin{aligned}
= & -\left.u \mu \frac{d v}{d z}\right|_{0} ^{h_{\mathrm{O}}}+\int_{0}^{h_{\mathrm{O}}} u d\left[\mu \frac{d v}{d z}\right]+\langle q v, u\rangle \\
= & -u\left(h_{\mathrm{O}}\right) \mu\left(h_{\mathrm{O}}\right) \frac{d v}{d z}\left(h_{\mathrm{O}}\right)+u(0) \mu(0) \frac{d v}{d z}(0) \\
& +\int_{0}^{h_{\mathrm{O}}} u \frac{d}{d z}\left(\mu \frac{d v}{d z}\right)+\langle q v, u\rangle \\
= & \int_{0}^{h_{\mathrm{O}}} u \frac{d}{d z}\left(\mu \frac{d v}{d z}\right)+\langle q v, u\rangle \\
= & \int_{0}^{h_{\mathrm{O}}} u\left[\frac{1}{\mu} \frac{d}{d z}\left(\mu \frac{d v}{d z}\right)+v \frac{d}{d z}\left(\frac{1}{\mu} \frac{d \mu}{d z}\right)+k^{2} v\right] \mu(z) d z \\
= & \int_{0}^{h_{\mathrm{O}}}\left[\frac{d^{2} v}{d z^{2}}+\frac{d}{d z}\left\{\frac{1}{\mu} \frac{d \mu}{d z} v\right\}+k^{2} v\right] u \mu(z) d z \\
= & \left\langle L_{\mathrm{TE}}^{a} v, u\right\rangle .
\end{aligned}
$$

From (43), the adjoint form of the operator can be identified as

$$
L_{\mathrm{TE}}^{a}=L_{\mathrm{TE}}
$$

Thus, it is seen that the operator is self-adjoint. Equation (44) was obtained by using a number of boundary conditions, which will be briefly covered in this section. Electric-wall conditions at $z=0, h_{\mathrm{O}}$ require transverse-field components $E_{r}$ and $E_{\phi}$ to be zero. From the $E_{\phi}$-field component expression in (22), one observes that pure Dirichlet conditions hold at the ends of the inner product domain. Coefficient $s_{j}$, from the first piece of $E_{\phi}$ generating the TE mode, indexed for $j z$-directed modes, has no $i k_{z}$ factor, implying

$$
\begin{aligned}
v(0) & =0 \\
v\left(h_{\mathrm{O}}\right) & =0 .
\end{aligned}
$$

The same conditions hold for $u$. Because within a region of a zone, $\mu(z)$ is stipulated to vary continuously, and for the zone to vary, at most, piecewise continuously, because the discontinuities only occur at the region interfaces, $d \mu / d z$ will be well behaved at the domain ends. Furthermore, requiring $\mu(z)$ to be constant within a region makes a Neumann condition also hold for it. Thus

$$
\begin{gathered}
\frac{d \mu}{d z}(0)=\left.\frac{d \mu}{d z}\right|_{z=0}=0 \\
\frac{d \mu}{d z}\left(h_{\mathrm{O}}\right)=\left.\frac{d \mu}{d z}\right|_{z=h_{\mathrm{O}}}=0 .
\end{gathered}
$$

Although (46) holds, conditions on $d \mu(z) / d z$ are not needed for obtaining the self-adjoint relation (43). This is like the case for the TM mode, where (24) was not required.

\section{Eigenvalues $\sigma_{m_{e}}$ of $\mathrm{TM}_{z}$}

Eigenvalues $\sigma_{m_{e}}$ of the $\mathrm{TM}_{z}$ modes, and the consequent values of $\lambda_{m_{e}}^{e}$, can be found by applying the boundary conditions on the electric-field component

$$
\begin{array}{ll}
E_{\phi}^{e \mathrm{O}}(r, \phi, z)=0, & z=h_{\mathrm{O}} \\
E_{\phi}^{e \mathrm{O}}(r, \phi, z)=0, & z=0
\end{array}
$$

and continuity conditions on the azimuthal components of the electric and magnetics fields

$$
\begin{aligned}
& \left.E_{\phi}^{e \mathrm{O}}(r, \phi, z)\right|_{h_{\mathrm{C}}+}=\left.E_{\phi}^{e \mathrm{O}}(r, \phi, z)\right|_{h_{\mathrm{C}}-} \\
& \left.H_{\phi}^{e \mathrm{O}}(r, \phi, z)\right|_{h_{\mathrm{C}}+}=\left.H^{e \mathrm{O}}(r, \phi, z)\right|_{h_{\mathrm{C}}-}
\end{aligned}
$$

$E_{\phi}^{e \mathrm{O}}$ can be written using the third-line form of (9), and (22):

$$
\begin{aligned}
E_{\phi}^{e \mathrm{O}}(r, \phi, z)= & \sum_{n=-\infty}^{\infty} \sum_{k_{z}=b} \frac{i p_{k_{z}}^{\mathrm{O}} n}{r} R_{n k_{z}}^{e}(r) \\
& \cdot\left[e^{i k_{z} z} A_{n k_{z}}^{e \mathrm{O}-}-e^{-i k_{z} z} A_{n k_{z}}^{e \mathrm{O}+}\right] e^{i n \phi}
\end{aligned}
$$

where

$$
\begin{aligned}
p_{j} & =\frac{i k_{z j}}{k_{d}^{2}-k_{z j}^{2}} \\
s_{j} & =-\frac{i \omega \mu_{d}}{k_{d}^{2}-k_{z j}^{2}} \\
u_{j} & =\frac{i \omega \varepsilon_{d}}{k_{d}^{2}-k_{z j}^{2}} .
\end{aligned}
$$

The comparable $H_{\phi}^{\mathrm{O}}$ expression to (22) is [10]

$$
\begin{aligned}
H_{\phi j}^{\mathrm{O}}= & \frac{p_{j}}{r} \frac{\partial H_{z j}^{\mathrm{O}}}{\partial \phi}-u_{j} \frac{\partial E_{z j}^{\mathrm{O}}}{\partial r} \\
H_{\phi}^{e \mathrm{O}}(r, \phi, z)= & \sum_{n=-\infty}^{\infty} \sum_{k_{z}=b}-u_{k_{z}}^{\mathrm{O}} \frac{d R_{n k_{z}}^{e}(r)}{d r} \\
& \cdot\left[e^{i k_{z} z} A_{n k_{z}}^{e \mathrm{O}-}+e^{-i k_{z} z} A_{n k_{z}}^{e \mathrm{O}+}\right] e^{i n \phi} .
\end{aligned}
$$

Electric-wall boundary conditions (47) and (48) yield with the help of (51)

$$
\begin{array}{r}
e^{i k_{z}^{\mathrm{OT}} h_{\mathrm{O}}} A_{n m_{e}}^{e \mathrm{OT}-}-e^{-i k_{z}^{\mathrm{OT}} h_{\mathrm{O}}} A_{n m_{e}}^{e \mathrm{OT}+}=0 \\
e^{i k_{z}^{\mathrm{OB}} \cdot 0} A_{n m_{e}}^{e \mathrm{OB}-}-e^{-i k_{z}^{\mathrm{OB}} \cdot \mathrm{o}} A_{n m_{e}}^{e \mathrm{OB}+}=0 .
\end{array}
$$

These conditions have resulted from the global nature of the radial $\sigma_{m_{e}}$ propagation constant or $m_{e}$ index, allowing the radial function and factors depending only on this index to drop out of the equations. Superscripts " $T$ " and " $B$ " denote, respectively, the top and bottom regions in the outside zone. It should be realized here that $k_{z}$ has an implicit dependence on $m_{e}$ so that when we see the perpendicular propagation constant, it is understood that it constitutes an abbreviation for $k_{z m_{e}}^{\mathrm{OT}}$ (for the top region, for example).

Continuity conditions (49) and (50) become, using, respectively, (51) and (54)

$$
\begin{gathered}
{\left[e^{i k_{z}^{\mathrm{OT}} h_{\mathrm{C}}} A_{n m_{e}}^{e \mathrm{OT}-}-e^{-i k_{z}^{\mathrm{OT}} h_{\mathrm{C}}} A_{n m_{e}}^{e \mathrm{OT}+}\right] k_{z}^{\mathrm{OT}}} \\
=\left[e^{i k_{z}^{\mathrm{OB}} h_{\mathrm{C}}} A_{n m_{e}}^{e \mathrm{OB}-}-e^{-i k_{z}^{\mathrm{OB}} h_{\mathrm{C}}} A_{n m_{e}}^{e \mathrm{OB}+}\right] k_{z}^{\mathrm{OB}} \\
{\left[e^{i k_{z}^{\mathrm{OT}} h_{\mathrm{C}}} A_{n m_{e}}^{e \mathrm{OT}-}+e^{-i k_{z}^{\mathrm{OT}} h_{\mathrm{C}}} A_{n m_{e}}^{e \mathrm{OT}+}\right] \varepsilon_{d}^{\mathrm{OT}}} \\
=\left[e^{i k_{z}^{\mathrm{OB}} h_{\mathrm{C}}} A_{n m_{e}}^{e \mathrm{OB}-}+e^{-i k_{z}^{\mathrm{OB}} h_{\mathrm{C}}} A_{n m_{e}}^{e \mathrm{OB}+}\right] \varepsilon_{d}^{\mathrm{OB}} .
\end{gathered}
$$

Adding and subtracting these two equations from each other and utilizing (56) gives the top-region amplitude coefficients

$$
\begin{aligned}
A_{n m_{e}}^{e \mathrm{OT}-}=\frac{1}{2} e^{-i k_{z}^{\mathrm{OT}} h_{\mathrm{C}}}\left\{e^{i k_{z}^{\mathrm{OB}} h_{\mathrm{C}}}\left[\frac{\varepsilon_{d}^{\mathrm{OB}}}{\varepsilon_{d}^{\mathrm{OT}}}+\frac{k_{z}^{\mathrm{OB}}}{k_{z}^{\mathrm{OT}}}\right]\right. \\
\left.+e^{-i k_{z}^{\mathrm{OB}} h_{\mathrm{C}}}\left[\frac{\varepsilon_{d}^{\mathrm{OB}}}{\varepsilon_{d}^{\mathrm{OT}}}-\frac{k_{z}^{\mathrm{OB}}}{k_{z}^{\mathrm{OT}}}\right]\right\} A_{n m_{e}}^{e \mathrm{OB}-}
\end{aligned}
$$




$$
\begin{aligned}
A_{n m_{e}}^{e \mathrm{OT}+}=\frac{1}{2} e^{i k_{z}^{\mathrm{OT}} h_{\mathrm{C}}\{}\left\{e^{i k_{z}^{\mathrm{OB}} h_{\mathrm{C}}}\left[\frac{\varepsilon_{d}^{\mathrm{OB}}}{\varepsilon_{d}^{\mathrm{OT}}}-\frac{k_{z}^{\mathrm{OB}}}{k_{z}^{\mathrm{OT}}}\right]\right. \\
\\
\left.+e^{-i k_{z}^{\mathrm{OB}} h_{\mathrm{C}}}\left[\frac{\varepsilon_{d}^{\mathrm{OB}}}{\varepsilon_{d}^{\mathrm{OT}}}+\frac{k_{z}^{\mathrm{OB}}}{k_{z}^{\mathrm{OT}}}\right]\right\} A_{n m_{e}}^{e \mathrm{OB}-}
\end{aligned}
$$

It is also helpful to define a ratio of the two amplitudes

$$
R_{e}^{\mathrm{OT}}=\frac{A_{n m_{e}}^{e \mathrm{OT}+}}{A_{n m_{e}}^{e \mathrm{OT}-}}
$$

so that

$$
\begin{aligned}
& R_{e}^{\mathrm{OT}}=e^{2 i k_{z}^{\mathrm{OT}}} h_{\mathrm{C}} \\
& \cdot \frac{e^{i k_{z}^{\mathrm{OB}} h_{\mathrm{C}}}\left[\frac{\varepsilon_{d}^{\mathrm{OB}}}{\varepsilon_{d}^{\mathrm{OT}}}-\frac{k_{z}^{\mathrm{OB}}}{k_{z}^{\mathrm{OT}}}\right]+e^{-i k_{z}^{\mathrm{OB}} h_{\mathrm{C}}}\left[\frac{\varepsilon_{d}^{\mathrm{OB}}}{\varepsilon_{d}^{\mathrm{OT}}}+\frac{k_{z}^{\mathrm{OB}}}{k_{z}^{\mathrm{OT}}}\right]}{e^{i k_{z}^{\mathrm{OB}} h_{\mathrm{C}}}\left[\frac{\varepsilon_{d}^{\mathrm{OB}}}{\varepsilon_{d}^{\mathrm{OT}}}+\frac{k_{z}^{\mathrm{OB}}}{k_{z}^{\mathrm{OT}}}\right]+e^{-i k_{z}^{\mathrm{OB}} h_{\mathrm{C}}}\left[\frac{\varepsilon_{d}^{\mathrm{OB}}}{\varepsilon_{d}^{\mathrm{OT}}}-\frac{k_{z}^{\mathrm{OB}}}{k_{z}^{\mathrm{OT}}}\right]} .
\end{aligned}
$$

Similarly, referring back to (56)

$$
R_{e}^{\mathrm{OB}}=\frac{A_{n m_{e}+}^{e \mathrm{OB}+}}{A_{n m_{e}}^{e \mathrm{OB}-}}=1 .
$$

Insert amplitude relations (59) and (60) into the electric-wall condition (55) to find the characteristic eigenvalue equation

$$
\begin{gathered}
e^{i k_{z}^{\mathrm{OT}}\left(h_{\mathrm{O}}-h_{\mathrm{C}}\right)}\left\{e^{-i k_{z}^{\mathrm{OB}} h_{\mathrm{C}}}\left[\frac{\varepsilon_{d}^{\mathrm{OB}}}{\varepsilon_{d}^{\mathrm{OT}}}+\frac{k_{z}^{\mathrm{OB}}}{k_{z}^{\mathrm{OT}}}\right]\right. \\
\left.+e^{-i k_{z}^{\mathrm{OB}} h_{\mathrm{C}}}\left[\frac{\varepsilon_{d}^{\mathrm{OB}}}{\varepsilon_{d}^{\mathrm{OT}}}-\frac{k_{z}^{\mathrm{OB}}}{k_{z}^{\mathrm{OT}}}\right]\right\} \\
-e^{-i k_{z}^{\mathrm{OT}}\left(h_{\mathrm{O}}-h_{\mathrm{C}}\right)}\left\{e^{i k_{z}^{\mathrm{OB}} h_{\mathrm{C}}}\left[\frac{\varepsilon_{d}^{\mathrm{OB}}}{\varepsilon_{d}^{\mathrm{OT}}}-\frac{k_{z}^{\mathrm{OB}}}{k_{z}^{\mathrm{OT}}}\right]\right. \\
\left.+e^{-i k_{z}^{\mathrm{OB}}} h_{\mathrm{C}}\left[\frac{\varepsilon_{d}^{\mathrm{OB}}}{\varepsilon_{d}^{\mathrm{OT}}}+\frac{k_{z}^{\mathrm{OB}}}{k_{z}^{\mathrm{OT}}}\right]\right\}=0 .
\end{gathered}
$$

Keeping the bracketed groupings in (64), identifying $\theta_{1}=$ $k_{z}^{\mathrm{OT}} h_{\mathrm{I}}+k_{z}^{\mathrm{OB}} h_{\mathrm{C}}$ and $\theta_{2}=k_{z}^{\mathrm{OT}} h_{\mathrm{I}}-k_{z}^{\mathrm{OB}} h_{\mathrm{C}}$, the exponential transcendental eigenvalue equation can be converted to the following trigonometric form:

$$
\frac{\sin \left[k_{z}^{\mathrm{OT}} h_{\mathrm{I}}+k_{z}^{\mathrm{OB}} h_{\mathrm{C}}\right]}{\sin \left[k_{z}^{\mathrm{OT}} h_{\mathrm{I}}-k_{z}^{\mathrm{OB}} h_{\mathrm{C}}\right]}=\frac{\frac{k_{z}^{\mathrm{OB}}}{k_{z}^{\mathrm{OT}}}-\frac{\varepsilon_{d}^{\mathrm{OB}}}{\varepsilon_{d}^{\mathrm{OT}}}}{\frac{k_{z}^{\mathrm{OB}}}{k_{z}^{\mathrm{OT}}}+\frac{\varepsilon_{d}^{\mathrm{OB}}}{\varepsilon_{d}^{\mathrm{OT}}}} .
$$

Here, $h_{\mathrm{I}}=h_{\mathrm{O}}-h_{\mathrm{C}}$. Note that for the limiting case where the top and bottom regions become identical, the numerator becomes zero and (65) reduces to the familiar form $\sin \left[k_{k}^{\mathrm{O}} h_{\mathrm{O}}\right]=0$. Another useful form may be obtained by grouping according to the ratios $k_{z}^{\mathrm{OB}} / k_{z}^{\mathrm{OT}}$ and $\varepsilon_{d}^{\mathrm{OB}} / \varepsilon_{d}^{\mathrm{OT}}$. Again, the exponential transcendental eigenvalue equation is changed into a trigonometric expression, which can be set down in the following two ways:

$$
\begin{aligned}
& \frac{\varepsilon_{d}^{\mathrm{OB}}}{\varepsilon_{d}^{\mathrm{OT}}} \tan \left[k_{z}^{\mathrm{OT}} h_{\mathrm{I}}\right]=-\frac{k_{z}^{\mathrm{OB}}}{k_{z}^{\mathrm{OT}}} \tan \left[k_{z}^{\mathrm{OB}} h_{\mathrm{C}}\right] \\
& \frac{k_{z}^{\mathrm{OT}}}{\varepsilon_{d}^{\mathrm{OT}}} \tan \left[k_{z}^{\mathrm{OT}} h_{\mathrm{I}}\right]=-\frac{k_{z}^{\mathrm{OB}}}{\varepsilon_{d}^{\mathrm{OB}}} \tan \left[k_{z}^{\mathrm{OB}} h_{\mathrm{C}}\right]
\end{aligned}
$$

The first form of this tangent relationship is written in terms of the propagation constant and dielectric ratios. The second form relates the top-region quantities to the bottom-region quantities. Equation (66b) can be shown to have the same form as indicated for a stacked inhomogeneous dielectric zone [6]. But we must be aware that now the material of the regions can be dielectric, magnetic, or of mixed permittivity and permeability character. The transcendental eigenvalue equation for the $\mathrm{TE}_{z}$ will be seen later to differ explicitly from the pure dielectric form even though the $\mathrm{TM}_{z}$ has not.

That (66) or (65) constitute eigenvalue equations for $\sigma_{m_{e}}^{\mathrm{O}}$ can be understood if the separation equations are found from the differential governing equation (13) for $Z_{m_{e}}^{e}$ with the help of (10) providing the exponential $Z_{m_{e}}^{e}$ form. Inserting (10) into (13) for the top and bottom regions in the zone, realizing that the inhomogeneous dielectric constant term drops out inside each region, yields

$$
\begin{aligned}
& -\left(k_{z}^{\mathrm{OT}}\right)^{2}+\left[\left(k_{d}^{\mathrm{OT}}\right)^{2}+\lambda_{m_{e}}^{e}\right]=0 \\
& -\left(k_{z}^{\mathrm{OB}}\right)^{2}+\left[\left(k_{d}^{\mathrm{OB}}\right)^{2}+\lambda_{m_{e}}^{e}\right]=0 .
\end{aligned}
$$

Invoking (11) for the separation constant, and taking the positive branches in (67), gives

$$
\begin{aligned}
& k_{z}^{\mathrm{OT}}=\sqrt{\left(k_{d}^{\mathrm{OT}}\right)^{2}-\left(\sigma_{m_{e}}^{\mathrm{O}}\right)^{2}} \\
& k_{z}^{\mathrm{OB}}=\sqrt{\left(k_{d}^{\mathrm{OB}}\right)^{2}-\left(\sigma_{m_{e}}^{\mathrm{O}}\right)^{2}} .
\end{aligned}
$$

Inserting (68) into (66b) gives a single transcendental equation in terms of the unknown $\sigma_{m_{e}}^{\mathrm{O}}$. Material-region propagation constants are delineatable once (6) is examined:

$$
\begin{aligned}
& \left(k_{d}^{\mathrm{OT}}\right)^{2}=\omega^{2} \mu_{d}^{\mathrm{OT}} \varepsilon_{d}^{\mathrm{OT}} \\
& \left(k_{d}^{\mathrm{OB}}\right)^{2}=\omega^{2} \mu_{d}^{\mathrm{OB}} \varepsilon_{d}^{\mathrm{OB}} .
\end{aligned}
$$

Return to the amplitude ratio $R_{e}^{\mathrm{OT}}$. Following the same reasoning in finding (66) using grouping by $k_{z}^{\mathrm{OB}} / k_{z}^{\mathrm{OT}}$ and $\varepsilon_{d}^{\mathrm{OB}} / \varepsilon_{d}^{\mathrm{OT}}$, yields (70) shown at the bottom of the page. Eliminating the bottom-region information in (70) by using the eigenvalue equation form (66b), the compact formula

$$
R_{e}^{\mathrm{OT}}=e^{2 i k_{z}^{\mathrm{OT}} h_{\mathrm{C}}} \frac{1+i \tan \left(k_{z}^{\mathrm{OT}} h_{\mathrm{I}}\right)}{1-i \tan \left(k_{z}^{\mathrm{OT}} h_{\mathrm{I}}\right)}
$$

results. Using ratio grouping and (66), the backward and forward amplitude coefficients in (59) and (60) for the top

$$
R_{e}^{\mathrm{OT}}=e^{2 i k_{z}^{\mathrm{OT}}} h_{\mathrm{C}} \frac{\left[\varepsilon_{d}^{\mathrm{OB}} / \varepsilon_{d}^{\mathrm{OT}}\right] \cos \left(k_{z}^{\mathrm{OB}} h_{\mathrm{C}}\right)-i\left[k_{z}^{\mathrm{OB}} / k_{z}^{\mathrm{OT}}\right] \sin \left(k_{z}^{\mathrm{OB}} h_{\mathrm{C}}\right)}{\left[\varepsilon_{d}^{\mathrm{OB}} / \varepsilon_{d}^{\mathrm{OT}}\right] \cos \left(k_{z}^{\mathrm{OB}} h_{\mathrm{C}}\right)+i\left[k_{z}^{\mathrm{OB}} / k_{z}^{\mathrm{OT}}\right] \sin \left(k_{z}^{\mathrm{OB}} h_{\mathrm{C}}\right)}
$$


region are now

$$
\begin{aligned}
A_{n m_{e}}^{e \mathrm{OT}-}= & \frac{\varepsilon_{d}^{\mathrm{OB}}}{\varepsilon_{d}^{\mathrm{OT}}} \cos \left(k_{z}^{\mathrm{OT}} h_{\mathrm{C}}\right) e^{-i k_{z}^{\mathrm{OT}} h_{\mathrm{C}}}\left\{1-i \tan \left(k_{z}^{\mathrm{OT}} h_{\mathrm{I}}\right)\right\} \\
& \cdot A_{n m_{e}}^{e \mathrm{OB}-} \\
A_{n m_{e}}^{e \mathrm{OT}+}= & \frac{\varepsilon_{d}^{\mathrm{OB}}}{\varepsilon_{d}^{\mathrm{OT}}} \cos \left(k_{z}^{\mathrm{OT}} h_{\mathrm{C}}\right) e^{i k_{z}^{\mathrm{OT}} h_{\mathrm{C}}}\left\{1+i \tan \left(k_{z}^{\mathrm{OT}} h_{\mathrm{I}}\right)\right\} \\
& \cdot A_{n m_{e}}^{e \mathrm{OB}-} .
\end{aligned}
$$

\section{Eigenvalues $\sigma_{m_{h}}$ of $\mathrm{TE}_{z}$}

Eigenvalues $\sigma_{m_{h}}$ of the $\mathrm{TE}_{z}$ modes, and the consequent values of $\lambda_{m_{h}}^{h}$ can be found by applying the boundary conditions on the electric-field component

$$
\begin{array}{ll}
E_{\phi}^{h \mathrm{O}}(r, \phi, z)=0, & z=h_{\mathrm{O}} \\
E_{\phi}^{h \mathrm{O}}(r, \phi, z)=0, & z=0
\end{array}
$$

and continuity conditions on the azimuthal components of the electric and magnetics fields

$$
\begin{aligned}
& \left.E_{\phi}^{h \mathrm{O}}(r, \phi, z)\right|_{h_{\mathrm{C}}+}=\left.E_{\phi}^{h \mathrm{O}}(r, \phi, z)\right|_{h_{\mathrm{C}}-} \\
& \left.H_{\phi}^{h \mathrm{O}}(r, \phi, z)\right|_{h_{\mathrm{C}}+}=\left.H_{\phi}^{h \mathrm{O}}(r, \phi, z)\right|_{h_{\mathrm{C}}-}
\end{aligned}
$$

$E_{\phi}^{h \mathrm{O}}$ can be written using the third-line form of (34) and (22) as follows:

$$
\begin{aligned}
E_{\phi}^{h \mathrm{O}}(r, \phi, z)= & \sum_{n=-\infty}^{\infty} \sum_{k_{z}=b}-s_{k_{z}}^{\mathrm{O}} \frac{d R_{n k_{z}}^{e}(r)}{d r} \\
& \cdot\left[e^{i k_{z} z} A_{n k_{z}}^{h \mathrm{O}-}+e^{-i k_{z} z} A_{n k_{z}}^{h \mathrm{O}+}\right] e^{i n \phi} .
\end{aligned}
$$

$H_{\phi}^{h \mathrm{O}}$ can be expressed using the first part of (53) as

$$
\begin{aligned}
H_{\phi}^{h \mathrm{O}}(r, \phi, z)= & \sum_{n=-\infty}^{\infty} \sum_{k_{z}=b} \frac{i p_{k_{z}}^{\mathrm{O}} n}{r} R_{n k_{z}}^{h}(r) \\
& \cdot\left[e^{i k_{z} z} A_{n k_{z}}^{h \mathrm{O}-}-e^{-i k_{z} z} A_{n k_{z}}^{h \mathrm{O}+}\right] e^{i n \phi} .
\end{aligned}
$$

Electric-wall boundary conditions (74) and (75) yield, with the help of (78)

$$
\begin{array}{r}
e^{i k_{z}^{\mathrm{OT}} h_{\mathrm{O}}} A_{n m_{e}}^{h \mathrm{OT}-}+e^{-i k_{z}^{\mathrm{OT}} h \mathrm{O}} A_{n m_{e}}^{h \mathrm{OT}+}=0 \\
e^{i k_{z}^{\mathrm{OB}} \cdot 0} A_{n m_{e}}^{h \mathrm{OB}-}+e^{-i k_{z}^{\mathrm{OB}} \cdot 0} A_{n m_{e}}^{h \mathrm{OB}+}=0 .
\end{array}
$$

These conditions have resulted from the global nature of the radial $\sigma_{m_{h}}$ propagation constant or $m_{h}$ index, allowing the radial function and factors depending only on this index to drop out of the equations. It should be realized here that $k_{z}$ has an implicit dependence on $m_{h}$ so that when we see the perpendicular propagation constant, it is understood that it constitutes an abbreviation for $k_{z m_{h}}^{\mathrm{OT}}$ (e.g., for the top region).
Continuity conditions (76) and (77) become, using, respectively, (78) and (79)

$$
\begin{aligned}
& {\left[e^{i k_{z}^{\mathrm{OT}} h_{\mathrm{C}}} A_{n m_{h}}^{h \mathrm{OT}-}+e^{-i k_{z}^{\mathrm{OT}} h_{\mathrm{C}}} A_{n m_{h}}^{h \mathrm{OT}+}\right] \mu_{d}^{\mathrm{OT}}} \\
& \quad=\left[e^{i k_{z}^{\mathrm{OB}} h_{\mathrm{C}}} A_{n m_{h}}^{h \mathrm{OB}-}+e^{-i k_{z}^{\mathrm{OB}} h_{\mathrm{C}}} A_{n m_{h}}^{h \mathrm{OB}+}\right] \mu_{d}^{\mathrm{OB}} \\
& {\left[e^{i k_{z}^{\mathrm{OT}} h_{\mathrm{C}}} A_{n m_{h}}^{h \mathrm{OT}-}-e^{-i k_{z}^{\mathrm{OT}} h_{\mathrm{O}}} A_{n m_{h}}^{h \mathrm{OT}+}\right] k_{z}^{\mathrm{OT}}} \\
& \quad=\left[e^{i k_{z}^{\mathrm{OB}} h_{\mathrm{C}}} A_{n m_{h}}^{h \mathrm{OB}-}-e^{-k_{z}^{\mathrm{OB}} h_{\mathrm{C}}} A_{n m_{h}}^{h \mathrm{OB}+}\right] k_{z}^{\mathrm{OB}} .
\end{aligned}
$$

Adding and subtracting these two equations from each other, and utilizing (81), gives the top-region amplitude coefficients

$$
\begin{aligned}
& A_{n m_{h}}^{h \mathrm{OT}-}=\frac{1}{2} e^{-i k_{z}^{\mathrm{OT}} h_{\mathrm{C}}}\left\{e^{i k_{z}^{\mathrm{OB}} h_{\mathrm{C}}}\left[\frac{\mu_{d}^{\mathrm{OB}}}{\mu_{d}^{\mathrm{OT}}}+\frac{k_{z}^{\mathrm{OB}}}{k_{z}^{\mathrm{OT}}}\right]\right. \\
& \left.-e^{i k_{z}^{\mathrm{OB}} h_{\mathrm{C}}}\left[\frac{\mu_{d}^{\mathrm{OB}}}{\mu_{d}^{\mathrm{OT}}}-\frac{k_{z}^{\mathrm{OB}}}{k_{z}^{\mathrm{OT}}}\right]\right\} A_{n m_{h}}^{h \mathrm{OB}-} \\
& A_{n m_{h}}^{h \mathrm{OT}+}=\frac{1}{2} e^{-i k_{z}^{\mathrm{OT}} h_{\mathrm{C}}}\left\{e^{i k_{z}^{\mathrm{OB}} h_{\mathrm{C}}}\left[\frac{\mu_{d}^{\mathrm{OB}}}{\mu_{d}^{\mathrm{OT}}}-\frac{k_{z}^{\mathrm{OB}}}{k_{z}^{\mathrm{OT}}}\right]\right. \\
& \left.-e^{-i k_{z}^{\mathrm{OB}} h_{\mathrm{C}}}\left[\frac{\mu_{d}^{\mathrm{OB}}}{\mu_{d}^{\mathrm{OT}}}+\frac{k_{z}^{\mathrm{OB}}}{k_{z}^{\mathrm{OT}}}\right]\right\} A_{n m_{h}}^{h \mathrm{OB}-} \text {. }
\end{aligned}
$$

Define a ratio of the backward and forward wave amplitudes

$$
R_{h}^{\mathrm{OT}}=\frac{A_{n m_{h}}^{h \mathrm{OT}+}}{A_{n m_{h}}^{h \mathrm{OT}-}}
$$

so that (87) results, shown at the bottom of the page. Similarly, referring back to (81)

$$
R_{h}^{\mathrm{OB}}=\frac{A_{n m_{h}}^{h \mathrm{OB}+}}{A_{n m_{h}}^{h \mathrm{OB}-}}=-1 .
$$

Insert amplitude relations (84) and (85) into the electric-wall condition (80) to find the characteristic eigenvalue equation

$$
\begin{aligned}
& e^{i k_{z}^{\mathrm{OT}}\left(h_{\mathrm{O}}-h_{\mathrm{C}}\right)}\left\{e^{i k_{z}^{\mathrm{OB}} h_{\mathrm{C}}}\left[\frac{\mu_{d}^{\mathrm{OB}}}{\mu_{d}^{\mathrm{OT}}}+\frac{k_{z}^{\mathrm{OB}}}{k_{z}^{\mathrm{OT}}}\right]\right. \\
& \left.-e^{-i k_{z}^{\mathrm{OB}} h_{\mathrm{C}}}\left[\frac{\mu_{d}^{\mathrm{OB}}}{\mu_{d}^{\mathrm{OT}}}-\frac{k_{z}^{\mathrm{OB}}}{k_{z}^{\mathrm{OT}}}\right]\right\} \\
& -e^{-i k_{z}^{\mathrm{OT}}\left(h_{\mathrm{O}}-h_{\mathrm{C}}\right)}\left\{e^{i k_{z}^{\mathrm{OB}} h_{\mathrm{C}}}\left[\frac{\mu_{d}^{\mathrm{OB}}}{\mu_{d}^{\mathrm{OT}}}-\frac{k_{z}^{\mathrm{OB}}}{k_{z}^{\mathrm{OT}}}\right]\right. \\
& \left.-e^{-i k_{z}^{\mathrm{OB}} h_{\mathrm{C}}}\left[\frac{\mu_{d}^{\mathrm{OB}}}{\mu_{d}^{\mathrm{OT}}}+\frac{k_{z}^{\mathrm{OB}}}{k_{z}^{\mathrm{OT}}}\right]\right\}=0 .
\end{aligned}
$$

Keeping the bracketed groupings in (89), identifying $\theta_{1}=$ $k_{z}^{\mathrm{OT}} h_{\mathrm{I}}+k_{z}^{\mathrm{OB}} h_{\mathrm{C}}$ and $\theta_{2}=k_{z}^{\mathrm{OT}} h_{\mathrm{I}}-k_{z}^{\mathrm{OB}} h_{\mathrm{C}}$, the exponential

$$
R_{h}^{\mathrm{OT}}=e^{2 i k_{z}^{\mathrm{OT}}} h_{\mathrm{C}} \frac{e^{i k_{z}^{\mathrm{OB}} h_{\mathrm{C}}}\left[\frac{\mu_{d}^{\mathrm{OB}}}{\mu_{d}^{\mathrm{OT}}}-\frac{k_{z}^{\mathrm{OB}}}{k_{z}^{\mathrm{OT}}}\right]-e^{-i k_{z}^{\mathrm{OB}} h_{\mathrm{C}}}\left[\frac{\mu_{d}^{\mathrm{OB}}}{\mu_{d}^{\mathrm{OT}}}+\frac{k_{z}^{\mathrm{OB}}}{k_{z}^{\mathrm{OT}}}\right]}{e^{i k_{z}^{\mathrm{OB}} h_{\mathrm{C}}}\left[\frac{\mu_{d}^{\mathrm{OB}}}{\mu_{d}^{\mathrm{OT}}}+\frac{k_{z}^{\mathrm{OB}}}{k_{z}^{\mathrm{OT}}}\right]-e^{-i k_{z}^{\mathrm{OB}} h_{\mathrm{C}}}\left[\frac{\mu_{d}^{\mathrm{OB}}}{\mu_{d}^{\mathrm{OT}}}-\frac{k_{z}^{\mathrm{OB}}}{k_{z}^{\mathrm{OT}}}\right]}
$$


transcendental eigenvalue equation can be converted to the following trigonometric form:

$$
\frac{\sin \left[k_{z}^{\mathrm{OT}} h_{\mathrm{I}}+k_{z}^{\mathrm{OB}} h_{\mathrm{C}}\right]}{\sin \left[k_{z}^{\mathrm{OT}} h_{\mathrm{I}}-k_{z}^{\mathrm{OB}} h_{\mathrm{C}}\right]}=\frac{\frac{\mu_{d}^{\mathrm{OB}}}{\mu_{d}^{\mathrm{OT}}}-\frac{k_{z}^{\mathrm{OB}}}{k_{z}^{\mathrm{OT}}}}{\frac{\mu_{z}^{\mathrm{OB}}}{\mu_{d}^{\mathrm{OT}}}+\frac{k_{z}^{\mathrm{OB}}}{k_{z}^{\mathrm{OT}}}} .
$$

For the limiting case where the top and bottom regions become identical, the numerator becomes 0 and (90) reduces to the familiar form $\sin \left[k_{z}^{\mathrm{O}} h_{\mathrm{O}}\right]=0$. Another useful form may be obtained by grouping according to the propagation constant and permeability ratios $k_{z}^{\mathrm{OB}} / k_{z}^{\mathrm{OT}}$ and $\mu_{d}^{\mathrm{OB}} / \mu_{d}^{\mathrm{OT}}$. Again, the exponential transcendental eigenvalue equation is changed into a trigonometric expression, which can be set down in two ways as follows:

$$
\begin{aligned}
& \frac{\mu_{d}^{\mathrm{OT}}}{\mu_{d}^{\mathrm{OB}}} \tan \left[k_{z}^{\mathrm{OT}} h_{\mathrm{I}}\right]=-\frac{k_{z}^{\mathrm{OT}}}{k_{z}^{\mathrm{OB}}} \tan \left[k_{z}^{\mathrm{OB}} h_{\mathrm{C}}\right] \\
& \frac{\mu_{d}^{\mathrm{OT}}}{k_{z}^{\mathrm{OT}}} \tan \left[k_{z}^{\mathrm{OT}} h_{\mathrm{I}}\right]=-\frac{\mu_{d}^{\mathrm{OB}}}{k_{z}^{\mathrm{OB}}} \tan \left[k_{z}^{\mathrm{OB}} h_{\mathrm{C}}\right] .
\end{aligned}
$$

The first form of this tangent relationship is written in terms of the propagation constant and dielectric ratios. The second form relates the top-region quantities to the bottom-region quantities. Equation (91b) shows that the magnetic differences between regions in a zone appear explicitly in the eigenvalue equation for the $\mathrm{TE}_{z}$ modes, whereas $(66 \mathrm{~b})$ demonstrated that the dielectric differences between regions in a zone appear explicitly in the eigenvalue equation for the $\mathrm{TM}_{z}$ modes.

That (91) or (90) constitute eigenvalue equations for $\sigma_{m_{h}}^{\mathrm{O}}$ can be understood if the separation equations are found from the differential governing equation (38) for $Z_{m_{h_{h}}}^{h}$ with the help of (35) providing the exponential $Z_{m_{h}}^{h}$ form. Inserting (35) into (38) for the top and bottom regions in the zone, realizing that the inhomogeneous permeability term drops out inside each region, yields

$$
\begin{aligned}
& -\left(k_{z}^{\mathrm{OT}}\right)^{2}+\left[\left(k_{d}^{\mathrm{OT}}\right)^{2}+\lambda_{m_{h}}^{h}\right]=0 \\
& -\left(k_{z}^{\mathrm{OB}}\right)^{2}+\left[\left(k_{d}^{\mathrm{OB}}\right)^{2}+\lambda_{m_{h}}^{h}\right]=0 .
\end{aligned}
$$

Invoking (36) for the separation constant, and taking the positive branches in (92), gives

$$
\begin{aligned}
& k_{z}^{\mathrm{OT}}=\sqrt{\left(k_{d}^{\mathrm{OT}}\right)^{2}-\left(\sigma_{m_{h}}^{\mathrm{O}}\right)^{2}} \\
& k_{z}^{\mathrm{OB}}=\sqrt{\left(k_{d}^{\mathrm{OB}}\right)^{2}-\left(\sigma_{m_{h}}^{\mathrm{O}}\right)^{2}} .
\end{aligned}
$$

Inserting (93) into (91b) gives a single transcendental equation in terms of the unknown $\sigma_{m_{h}}^{\mathrm{O}}$.

Looking at the amplitude ratio $R_{h}^{\mathrm{OT}}$ in (87) again, using the same procedure in finding (91) using grouping by $k_{z}^{\mathrm{OB}} / k_{z}^{\mathrm{OT}}$ and $\mu_{d}^{\mathrm{OB}} / \mu_{d}^{\mathrm{OT}}$

$$
\begin{aligned}
R_{h}^{\mathrm{OT}}= & e^{2 i k_{z}^{\mathrm{OT}} h_{\mathrm{C}}} \\
& \cdot \frac{i\left[\mu_{d}^{\mathrm{OB}} / \mu_{d}^{\mathrm{OT}}\right] \sin \left(k_{z}^{\mathrm{OB}} h_{\mathrm{C}}\right)-\left[k_{z}^{\mathrm{OB}} / k_{z}^{\mathrm{OT}}\right] \cos \left(k_{z}^{\mathrm{OB}} h_{\mathrm{C}}\right)}{i\left[\mu_{d}^{\mathrm{OB}} / \mu_{d}^{\mathrm{OT}}\right] \sin \left(k_{z}^{\mathrm{OB}} h_{\mathrm{C}}\right)+\left[k_{z}^{\mathrm{OB}} / k_{z}^{\mathrm{OT}}\right] \cos \left(k_{z}^{\mathrm{OB}} h_{\mathrm{C}}\right)} .
\end{aligned}
$$

Eliminating the bottom-region information in (94) by using the eigenvalue equation form (91b), the compact formula

$$
R_{h}^{\mathrm{OT}}=-e^{2 i k_{z}^{\mathrm{OT}}} h_{\mathrm{C}} \frac{1+i \tan \left(k_{z}^{\mathrm{OT}} h_{\mathrm{I}}\right)}{1-i \tan \left(k_{z}^{\mathrm{OT}} h_{\mathrm{I}}\right)}
$$

results. Using ratio grouping and (91), the backward and forward amplitude coefficients in (84) and (85) for the top region are now

$$
\begin{aligned}
A_{n m_{h}}^{h \mathrm{OT}-}= & -\frac{\mu_{d}^{\mathrm{OB}}}{\mu_{d}^{\mathrm{OT}}} \frac{\sin \left(k_{z}^{\mathrm{OT}} h_{\mathrm{C}}\right)}{\tan \left(k_{z}^{\mathrm{OT}} h_{\mathrm{I}}\right)} e^{-i k_{z}^{\mathrm{OT}} h_{\mathrm{C}}}\left\{1-i \tan \left(k_{z}^{\mathrm{OT}} h_{\mathrm{I}}\right)\right\} \\
& \cdot A_{n m_{h}}^{h \mathrm{OB}-} \\
A_{n m_{h}}^{h \mathrm{OT}+}= & -\frac{\mu_{d}^{\mathrm{OB}}}{\mu_{d}^{\mathrm{OT}}} \frac{\sin \left(k_{z}^{\mathrm{OT}} h_{\mathrm{C}}\right)}{\tan \left(k_{z}^{\mathrm{OT}} h_{\mathrm{I}}\right)} e^{-i k_{z}^{\mathrm{OT}} h_{\mathrm{C}}}\left\{1+i \tan \left(k_{z}^{\mathrm{OT}} h_{\mathrm{I}}\right)\right\} \\
& \cdot A_{n m_{h}}^{h \mathrm{OB}-} .
\end{aligned}
$$

\section{E. Eigenvectors of $\mathrm{TM}_{z}$}

Return to (10), extracting out the third exponential wave form. Factor out the backward wave

$$
Z_{m_{e}}^{e}(z)=\left[e^{i k_{z}^{\mathrm{O}} z}+e^{-i k_{z}^{\mathrm{O}} z} \frac{A_{n k_{z}}^{e \mathrm{O}+}}{A_{n k_{z}}^{e \mathrm{O}-}}\right] A_{n m_{e}}^{e \mathrm{O}-}
$$

Writing (98) in terms of the different regions (two here), using the definitions (61) and (63)

$$
\begin{aligned}
Z_{m_{e}}^{e}(z) & \begin{cases}{\left[e^{i k_{z}^{\mathrm{OT}} z}+e^{-i k_{z}^{\mathrm{OT}} z} R_{e}^{\mathrm{OT}}\right] A_{n m_{e}}^{e \mathrm{OT}-}, \quad h_{\mathrm{C}} \leq z \leq h_{\mathrm{O}}} \\
{\left[e^{i k_{z}^{\mathrm{OB}} z}+e^{-i k_{z}^{\mathrm{OB}} z} R_{e}^{\mathrm{OB}}\right] A_{n m_{e}}^{e \mathrm{OB}-}, \quad 0 \leq z \leq h_{\mathrm{C}}}\end{cases} \\
= & \begin{cases}{\left[e^{i k_{z}^{\mathrm{OT}} z}+e^{-i k_{z}^{\mathrm{OT}} z} R_{e}^{\mathrm{OT}}\right] A_{n m_{e}}^{e \mathrm{OT}-},} & h_{\mathrm{C}} \leq z \leq h_{\mathrm{O}} \\
2 \cos \left(k_{z}^{\mathrm{OB}} z\right) A_{n m_{e}}^{e \mathrm{OB}-}, & 0 \leq z \leq h_{\mathrm{C}}\end{cases} \\
= & \left\{\begin{array}{ll}
{\left[e^{i k_{z}^{\mathrm{OT}} z}+e^{-i k_{z}^{\mathrm{OT}} z} R_{e}^{\mathrm{OT}}\right] R_{m_{e}}^{\mathrm{TB}-},} & h_{\mathrm{C}} \leq z \leq h_{\mathrm{O}} \\
2 \cos \left(k_{z}^{\mathrm{OB}} z\right), & 0 \leq z \leq h_{\mathrm{C}}
\end{array}\right\} \\
= & \bar{Z}_{m_{e} A_{n m_{e}}^{e \mathrm{OB}-}}^{A_{n m_{e}}} \\
= & \left\{\begin{array}{ll}
{\left[e^{i k_{z}^{\mathrm{OT}} z}+e^{-i k_{z}^{\mathrm{OT}} z} R_{e}^{\mathrm{OT}}\right],} & h_{\mathrm{C}} \leq z \leq h_{\mathrm{O}} \\
2 \cos \left(k_{z}^{\mathrm{OB}} z\right) R_{m_{e}}^{\mathrm{BT}-}, & 0 \leq z \leq h_{\mathrm{C}}
\end{array}\right\} A_{n m_{e}}^{e \mathrm{OT}-} \\
= & \overline{\bar{Z}}_{m_{e} A_{n m_{e}}^{e \mathrm{OT}-} .}
\end{aligned}
$$

The fourth and sixth forms of (99) created the scaled formulas for the eigenfunctions, separated from the unknown coefficients found in $Z_{m_{e}}^{e}(z)$. Known ratios

$$
R_{m_{e}}^{\mathrm{TB}-}=\left(R_{m_{e}}^{\mathrm{BT}-}\right)^{-1}=\frac{A_{n m_{e}}^{e \mathrm{OT}-}}{A_{n m_{e}}^{e \mathrm{OB}-}}
$$

available from (59), enable the unknown fields to be written in terms of one unknown amplitude for each zone (one external zone beyond the circulator in the current problem). The scaled forms are related to one another by

$$
\overline{\bar{Z}}_{m_{e}}^{e}=R_{m_{e}}^{\mathrm{BT}-\bar{Z}_{m_{e}}^{e}}
$$

Retrieving (71) and (72), the first scaled eigenfunction (the generator for the eigenvector) form in the top region can be 
evaluated, completing its explicit formula

$$
\bar{Z}_{m_{e}}^{e}=\left\{\begin{array}{lr}
2 \frac{\varepsilon_{d}^{\mathrm{OB}}}{\varepsilon_{d}^{\mathrm{OT}}} \frac{\cos \left(k_{z m_{e}}^{\mathrm{OB}} h_{\mathrm{C}}\right)}{\cos \left(k_{z m_{e}}^{\mathrm{OT}} h_{\mathrm{I}}\right)} \cos \left(k_{z m_{e}}^{\mathrm{OT}}\left[z-h_{\mathrm{O}}\right]\right), \\
2 \cos \left(k_{z m_{e}}^{\mathrm{OB}} z\right), & 0 \leq z \leq h_{\mathrm{O}}
\end{array}\right.
$$

One can easily show that (102) corresponds directly to a continuity condition at the $z=h_{\mathrm{C}}$ interface if it is multiplied by $\varepsilon(z)$, thereby satisfying the normal component of the displacement field continuity

$$
\left.D_{z}^{e \mathrm{O}}(r, \phi, z)\right|_{h_{\mathrm{C}}+}=\left.D_{z}^{e \mathrm{O}}(r, \phi, z)\right|_{h_{\mathrm{C}}-}
$$

Enlisting (99), the orthogonal property for $Z_{m_{e}}^{e}(z)$ given by (27) carries over to $\bar{Z}_{m_{e}}^{e}(z)$, i.e.,

$$
\left\langle Z_{m_{e}}^{e}(z), Z_{m_{e}^{\prime}}^{e}(z)\right\rangle=\left\langle\bar{Z}_{m_{e}}^{e}(z), \bar{Z}_{m_{e}^{\prime}}^{e}(z)\right\rangle=0 .
$$

It is instructive to verify that (102) indeed satisfies (104). Proceeding,

$$
\begin{aligned}
\left\langle\bar{Z}_{m_{e}}^{e}(z), \bar{Z}_{m_{e}^{\prime}}^{e}(z)\right\rangle & \\
= & \int_{0}^{h_{\mathrm{O}}} \bar{Z}_{m_{e}}^{e}(z) \bar{Z}_{m_{e}^{\prime}}^{e}(z) \varepsilon(z) d z \\
= & 4 \varepsilon_{d}^{\mathrm{OB}} \int_{0}^{h_{\mathrm{C}}} \cos \left(k_{z m_{e}}^{\mathrm{OB}} z\right) \cos \left(k_{z m_{e}^{\prime}}^{\mathrm{OB}} z\right) d z \\
& +4\left[\frac{\varepsilon_{d}^{\mathrm{OB}}}{\varepsilon_{d}^{\mathrm{OT}}}\right]^{2} \varepsilon_{d}^{\mathrm{OT}} \frac{\cos \left(k_{z m_{e}}^{\mathrm{OB}} h_{\mathrm{C}}\right)}{\cos \left(k_{z m_{e}}^{\mathrm{OT}} h_{\mathrm{I}}\right)} \frac{\cos \left(k_{z m_{e}^{\prime}}^{\mathrm{OB}} h_{\mathrm{C}}\right)}{\cos \left(k_{z m_{e}^{\prime}}^{\mathrm{OT}} h_{\mathrm{I}}\right)} \\
& \cdot \int_{h_{\mathrm{C}}}^{h_{\mathrm{O}}} \cos \left(k_{z m_{e}}^{\mathrm{OT}}\left[h_{\mathrm{O}}-z\right]\right) \cos \left(k_{z m_{e}^{\prime}}^{\mathrm{OT}}\left[h_{\mathrm{O}}-z\right]\right) d z .
\end{aligned}
$$

Referring to a compilation of integrals [11], the first definite integral in (105) is evaluated to be

$$
\begin{aligned}
& \left.\int_{0}^{h_{\mathrm{C}}} \cos \left(k_{z m_{e}}^{\mathrm{OB}} z\right) \cos k_{z m_{e}^{\prime}}^{\mathrm{OB}} z\right) d z \\
& =\frac{1}{\left(k_{z m_{e}}^{\mathrm{OB}}\right)^{2}-\left(k_{z m_{e}^{\prime}}^{\mathrm{OB}}\right)^{2}}\left[k_{z m_{e}}^{\mathrm{OB}} \sin \left(k_{z m_{e}}^{\mathrm{OB}} h_{\mathrm{C}}\right) \cos \left(k_{z m_{e}^{\prime}}^{\mathrm{OB}} h_{\mathrm{C}}\right)\right. \\
& \left.-k_{z m_{e}^{\prime}}^{\mathrm{OB}} \cos \left(k_{z m_{e}}^{\mathrm{OB}} h_{\mathrm{C}}\right) \sin \left(k_{z m_{e}^{\prime}}^{\mathrm{OB}} h_{\mathrm{C}}\right)\right] \text {. }
\end{aligned}
$$

Referencing the integration variable in the second integral to the $z=h_{\mathrm{O}}$ top plane, and making appropriate constant changes allows the use of (106) again. The difference between perpendicular propagation constant eigenvalues in the different regions, which act as a prefactors in both integrals, are found to be equal by (67) to $\left(\sigma_{m_{e}^{\prime}}^{\mathrm{O}}\right)^{2}-\left(\sigma_{m_{e}}^{\mathrm{O}}\right)^{2}$, reducing the inner product expression in (105) to

$$
\begin{aligned}
\left\langle\bar{Z}_{m_{e}}^{e}(z), \bar{Z}_{m_{e}^{\prime}}^{e}(z)\right\rangle & \\
= & \frac{4\left(\varepsilon_{d}^{\mathrm{OB}}\right)^{2}}{\left(\sigma_{m_{e}^{\prime}}^{\mathrm{O}}\right)^{2}-\left(\sigma_{m_{e}}^{\mathrm{O}}\right)^{2}} \\
& \cdot\left\{\frac{1}{\varepsilon_{d}^{\mathrm{OB}}}\left[k_{z m_{e}}^{\mathrm{OB}} \tan \left(k_{z m_{e}}^{\mathrm{OB}} h_{\mathrm{C}}\right)-k_{z m_{e}^{\prime}}^{\mathrm{OB}} \tan \left(k_{z m_{e}^{\prime}}^{\mathrm{OB}} h_{\mathrm{C}}\right)\right]\right. \\
& \left.\quad+\frac{1}{\varepsilon_{d}^{\mathrm{OT}}}\left[k_{z m_{e}}^{\mathrm{OT}} \tan \left(k_{z m_{e}}^{\mathrm{OT}} h_{\mathrm{I}}\right)-k_{z m_{e}^{\prime}}^{\mathrm{OT}} \tan \left(k_{z m_{e}^{\prime}}^{\mathrm{OT}} h_{\mathrm{I}}\right)\right]\right\} \\
& \times \cos \left(k_{z m_{e}}^{\mathrm{OB}} h_{\mathrm{C}}\right) \cos \left(h_{z m_{e}^{\prime}}^{\mathrm{OB}} h_{\mathrm{C}}\right) \\
= & 0
\end{aligned}
$$

invoking the eigenvalue equation (66). Thus, we have verified (104) directly.

The orthogonality relation may now be stated as

$$
\left\langle\bar{Z}_{m_{e}}^{e}(z), \bar{Z}_{m_{e}^{\prime}}^{e}(z)\right\rangle=C_{m_{e}}^{e} \delta_{m_{e}, m_{e}^{\prime}}
$$

Let us borrow the RHS of (105), and particularize to $m_{e}=m_{e}^{\prime}$ to obtain the square of the normalization constant which is equal to $C_{m_{e}}^{e}$ :

$$
\begin{aligned}
C_{m_{e}}^{e}= & 4 \varepsilon_{d}^{\mathrm{OB}} \int_{0}^{h_{\mathrm{C}}} \cos ^{2}\left(k_{z m_{e}}^{\mathrm{OB}} z\right) d z \\
& +4\left[\frac{\varepsilon_{d}^{\mathrm{OB}}}{\varepsilon_{d}^{\mathrm{OT}}}\right]^{2} \varepsilon_{d}^{\mathrm{OT}} \frac{\cos ^{2}\left(k_{z m_{e}}^{\mathrm{OB}} h_{\mathrm{C}}\right)}{\cos ^{2}\left(k_{z m_{e}}^{\mathrm{OT}} h_{\mathrm{I}}\right)} \\
& \cdot \int_{h_{\mathrm{C}}}^{h_{\mathrm{O}}} \cos ^{2}\left(k_{z m_{e}}^{\mathrm{OT}}\left[h_{\mathrm{O}}-z\right]\right) d z \\
= & 4 \varepsilon_{d}^{\mathrm{OB}}\left\{I_{1 e}+\frac{\varepsilon_{d}^{\mathrm{OB}}}{\varepsilon_{d}^{\mathrm{OT}}} \frac{\cos ^{2}\left(k_{z m_{e}}^{\mathrm{OB}} h_{\mathrm{C}}\right)}{\cos ^{2}\left(k_{z m_{e}}^{\mathrm{OT}} h_{\mathrm{I}}\right)} I_{2 e}\right\}
\end{aligned}
$$

where [11]

$$
\begin{aligned}
I_{1 e} & =\int_{0}^{h_{\mathrm{C}}} \cos ^{2}\left(k_{z m_{e}}^{\mathrm{OB}} z\right) d z \\
& =\frac{1}{k_{z m_{e}}^{\mathrm{OB}}}\left[\frac{1}{4} \sin \left(2 k_{z m_{e}}^{\mathrm{OB}} h_{\mathrm{C}}\right)+\frac{k_{z m_{e}}^{\mathrm{OB}} h_{\mathrm{C}}}{2}\right] \\
I_{2 e} & =\int_{h_{\mathrm{C}}}^{h_{\mathrm{O}}} \cos ^{2}\left(k_{z m_{e}}^{\mathrm{OT}}\left[h_{\mathrm{O}}-z\right]\right) d z \\
& =\frac{1}{k_{z m_{e}}^{\mathrm{OT}}}\left[\frac{1}{4} \sin \left(2 k_{z m_{e}}^{\mathrm{OT}} h_{\mathrm{I}}\right)+\frac{k_{z m_{e}}^{\mathrm{OT}} h_{\mathrm{I}}}{2}\right] .
\end{aligned}
$$

\section{F. Eigenvectors of $\mathrm{TE}_{z}$}

Go back to (35), extracting out the third exponential wave form. Factor out the backward wave

$$
Z_{m_{h}}^{h}(z)=\left[e^{i k_{z}^{\mathrm{O} z}}+e^{-i k_{z}^{\mathrm{O} z}} \frac{A_{n k_{z}}^{h \mathrm{O}+}}{A_{n k_{z}}^{h \mathrm{O}-}}\right] A_{n m_{h}}^{h \mathrm{O}-}
$$

Writing (110) in terms of the different regions (two here), using the definitions (86) and (88)

$$
\begin{aligned}
& Z_{m_{h}}^{h}(z) \\
& \quad= \begin{cases}{\left[e^{i k_{z}^{\mathrm{OT}} z}+e^{-i k_{z}^{\mathrm{OT}} z} R_{h}^{\mathrm{OT}}\right] A_{n m_{h}}^{h \mathrm{OT}-},} & h_{\mathrm{C}} \leq z \leq h_{\mathrm{O}} \\
{\left[e^{i k_{z}^{\mathrm{OB}} z}+e^{-i k_{z}^{\mathrm{OB}} z} R_{h}^{\mathrm{OB}}\right] A_{n m_{h}}^{h \mathrm{OB}-},} & 0 \leq z \leq h_{\mathrm{C}}\end{cases}
\end{aligned}
$$




$$
\begin{aligned}
& =\left\{\left[e^{i k_{z}^{\mathrm{OT}} z}+e^{-i k_{z}^{\mathrm{OT}} z} R_{h}^{\mathrm{OT}}\right] A_{n m_{h}}^{h \mathrm{OT}-}, \quad h_{\mathrm{C}} \leq z \leq h_{\mathrm{O}}\right. \\
& = \begin{cases}2 i \sin \left(k_{z}^{\mathrm{OB}} z\right) A_{n m_{h}}^{h \mathrm{OB}-}, & 0 \leq z \leq h_{\mathrm{C}}\end{cases} \\
& =\left\{\begin{array}{ll}
{\left[e^{i k_{z}^{\mathrm{OT}} z}+e^{-i k_{z}^{\mathrm{OT}} z} R_{h}^{\mathrm{OT}}\right] R_{m_{h}}^{\mathrm{TB}-},} & h_{\mathrm{C}} \leq z \leq h_{\mathrm{O}} \\
2 i \sin \left(k_{z}^{\mathrm{OB}} z\right), & 0 \leq z \leq h_{\mathrm{C}}
\end{array}\right\} \\
& \cdot A_{n m_{h}}^{h \mathrm{OB}-} \\
& =\bar{Z}_{m_{h}}^{h} A_{n m_{h}}^{h \mathrm{OB}-} \\
& =\left\{\begin{array}{ll}
{\left[e^{i k_{z}^{\mathrm{OT}} z}+e^{-i k_{z}^{\mathrm{OT}} z} R_{h}^{\mathrm{OT}}\right],} & h_{\mathrm{C}} \leq z \leq h_{\mathrm{O}} \\
2 i \sin \left(k_{z}^{\mathrm{OB}} z\right) R_{m_{h}}^{\mathrm{BT}-}, & 0 \leq z \leq h_{\mathrm{C}}
\end{array}\right\} A_{n m_{h}}^{h \mathrm{OT}-} \\
& =\overline{\bar{Z}}_{m_{h}}^{h} A_{n m_{h}}^{h \mathrm{OT}-} \text {. }
\end{aligned}
$$

The fourth and sixth forms of (111) created the scaled formulas for the eigenfunctions, separated from the unknown coefficients found in $Z_{m_{h}}^{h}(z)$. Known ratios

$$
R_{m_{h}}^{\mathrm{TB}-}=\left(R_{m_{h}}^{\mathrm{BT}-}\right)^{-1}=\frac{A_{n m_{h}}^{h \mathrm{OT}-}}{A_{n m_{h}}^{h \mathrm{OB}-}}
$$

available from (84), enable the unknown fields to be written in terms of one unknown amplitude for each zone. The scaled forms are related to one another by

$$
\overline{\bar{Z}}_{m_{h}}^{h}=R_{m_{h}}^{\mathrm{BT}-\bar{Z}_{m_{h}}^{h}} \text {. }
$$

Retrieving (95) and (96), the first scaled eigenfunction (the generator for the eigenvector) form in the top region can be evaluated, completing its explicit formula

$$
\bar{Z}_{m_{h}}^{h}=\left\{\begin{array}{cc}
-2 i \frac{\mu_{d}^{\mathrm{OB}}}{\mu_{d}^{\mathrm{OT}}} \frac{\sin \left(k_{z m_{h}}^{\mathrm{OB}} h_{\mathrm{C}}\right)}{\sin \left(k_{z m_{h}}^{\mathrm{OT}} h_{\mathrm{I}}\right)} \sin \left(k_{z m_{h}}^{\mathrm{OT}}\left[z-h_{\mathrm{O}}\right],\right. \\
2 i \sin \left(k_{z m_{h}}^{\mathrm{OB}} z\right), & h_{\mathrm{C}} \leq z \leq h_{\mathrm{O}}
\end{array}\right.
$$

Equation (114) can be shown to correspond directly to a continuity condition at the $z=h_{\mathrm{C}}$ interface if it is multiplied by $\mu(z)$, thereby satisfying the normal component of the $B$ field continuity

$$
\left.B_{z}^{h \mathrm{O}}(r, \phi, z)\right|_{h_{\mathrm{C}}+}=\left.B_{z}^{h \mathrm{O}}(r, \phi, z)\right|_{h_{\mathrm{C}}-} .
$$

Utilizing (111), the orthogonal property for $Z_{m_{h}}^{h}(z)$ given by (27) carries over to $\bar{Z}_{m_{h}}^{h}(z)$ when using the proper weight function in the inner product construction, i.e.,

$$
\left\langle Z_{m_{h}}^{h}(z), Z_{m_{h}^{\prime}}^{h}(z)\right\rangle=\left\langle\bar{Z}_{m_{h}}^{h}(z), \bar{Z}_{m_{h}^{\prime}}^{h}(z)\right\rangle=0 .
$$

It is also informative to verify that (114) does satisfy (116). Proceeding,

$$
\begin{aligned}
\left\langle\bar{Z}_{m_{h}}^{h}(z), \bar{Z}_{m_{h}^{\prime}}^{h}(z)\right\rangle & \\
= & \int_{0}^{h_{\mathrm{O}}} \bar{Z}_{m_{h}}^{h}(z) \bar{Z}_{m_{h}^{\prime}}^{h}(z) \mu(z) d z \\
= & -4 \mu_{d}^{\mathrm{OB}} \int_{0}^{h_{\mathrm{C}}} \sin \left(k_{z m_{h}}^{\mathrm{OB}} z\right) \sin \left(k_{z m_{h}^{\prime}}^{\mathrm{OB}} z\right) d z \\
& -4\left[\frac{\mu_{d}^{\mathrm{OB}}}{\mu_{d}^{\mathrm{OT}}}\right]^{2} \mu_{d}^{\mathrm{OT}} \frac{\sin \left(k_{z m_{h}}^{\mathrm{OB}} h_{\mathrm{C}}\right)}{\sin \left(k_{z m_{h}}^{\mathrm{OT}} h_{\mathrm{I}}\right)} \frac{\sin \left(k_{z m_{h}^{\prime}}^{\mathrm{OB}} h_{\mathrm{C}}\right)}{\sin \left(k_{z m_{h}^{\prime}}^{\mathrm{OT}} h_{\mathrm{I}}\right)} \\
& \cdot \int_{h_{\mathrm{C}}}^{h_{\mathrm{O}}} \sin \left(k_{z m_{h}}^{\mathrm{OT}}\left[h_{\mathrm{O}}-z\right]\right) \sin \left(k_{z m_{h}^{\prime}}^{\mathrm{OT}}\left[h_{\mathrm{O}}-z\right]\right) d z .
\end{aligned}
$$

Referring to a compilation of integrals [11], the first definite integral in (117) is evaluated to be

$$
\begin{aligned}
\int_{0}^{h_{\mathrm{C}}} & \sin \left(k_{z m_{h}}^{\mathrm{OB}} z\right) \sin \left(k_{z m_{h}^{\prime}}^{\mathrm{OB}} z\right) d z \\
= & \frac{1}{\left(k_{z m_{h}}^{\mathrm{OB}}\right)^{2}-\left(k_{z m_{h}^{\prime}}^{\mathrm{OB}}\right)^{2}}\left[k_{z m_{h}^{\prime}}^{\mathrm{OB}} \sin \left(k_{z m_{h}}^{\mathrm{OB}} h_{\mathrm{C}}\right)\right. \\
& \left.\cdot \cos \left(k_{z m_{h}^{\prime}}^{\mathrm{OB}} h_{\mathrm{C}}\right)-k_{z m_{h}}^{\mathrm{OB}} \cos \left(k_{z m_{h}}^{\mathrm{OB}} h_{\mathrm{C}}\right) \sin \left(k_{z m_{h}^{\prime}}^{\mathrm{OB}} h_{\mathrm{C}}\right)\right] .
\end{aligned}
$$

Referencing the integration variable in the second integral to the $z=h_{\mathrm{O}}$ top plane, and making appropriate constant changes allows the use of (118) again. The difference between perpendicular propagation constant eigenvalues in the different regions, which act as prefactors in both integrals, are found to be equal by (92) to $\left(\sigma_{m_{h^{\prime}}^{\prime}}^{\mathrm{O}}\right)^{2}-\left(\sigma_{m_{h}}^{\mathrm{O}}\right)^{2}$, reducing the inner product expression in (117) to

$$
\begin{aligned}
\left\langle\bar{Z}_{m_{h}}^{h}(z), \bar{Z}_{m_{h}^{\prime}}^{h}(z)\right\rangle & \\
= & \frac{-4\left(\mu_{d}^{\mathrm{OB}}\right)^{2}}{\left(\sigma_{m_{h}^{\prime}}^{\mathrm{O}}\right)^{2}-\left(\sigma_{m_{h}}^{\mathrm{O}}\right)^{2}} \\
& \cdot\left\{\frac{1}{\mu_{d}^{\mathrm{OB}}}\left[k_{z m_{h}^{\prime}}^{\mathrm{OB}} \cot \left(k_{z m_{h}^{\prime}}^{\mathrm{OB}} h_{\mathrm{C}}\right)-k_{z m_{h}}^{\mathrm{OB}} \cot \left(k_{z m_{h}}^{\mathrm{OB}} h_{\mathrm{C}}\right)\right]\right. \\
& \left.\quad+\frac{1}{\mu_{d}^{\mathrm{OT}}}\left[k_{z m_{h}^{\prime}}^{\mathrm{OT}} \cot \left(k_{z m_{h}^{\prime}}^{\mathrm{OT}} h_{\mathrm{I}}\right)-k_{z m_{h}}^{\mathrm{OT}} \cot \left(k_{z m_{h}}^{\mathrm{OT}} h_{\mathrm{I}}\right)\right]\right\} \\
& \quad \times \sin \left(k_{z m_{h}}^{\mathrm{OB}} h_{\mathrm{C}}\right) \sin \left(k_{z m_{h}^{\prime}}^{\mathrm{OB}} h_{\mathrm{C}}\right) \\
= & 0
\end{aligned}
$$

invoking the eigenvalue equation (91). Thus, we have verified (116) directly.

The orthogonality relation may now be stated as

$$
\left\langle\bar{Z}_{m_{h}}^{h}(z), \bar{Z}_{m_{h}^{\prime}}^{h}(z)\right\rangle=C_{m_{h}}^{h} \delta_{m_{h}, m_{h}^{\prime}} \cdot
$$

Let us borrow the RHS of (117), and particularize to $m_{h}=m_{h}^{\prime}$ to obtain the square of the normalization constant which is equal to $C_{m_{h}}^{h}$ :

$$
\begin{aligned}
C_{m_{h}}^{h}= & -4 \mu_{d}^{\mathrm{OB}} \int_{0}^{h_{\mathrm{C}}} \sin ^{2}\left(k_{z m_{e}}^{\mathrm{OB}} z\right) d z \\
& -4\left[\frac{\mu_{d}^{\mathrm{OB}}}{\mu_{d}^{\mathrm{OT}}}\right]^{2} \mu_{d}^{\mathrm{OT}} \frac{\sin ^{2}\left(k_{z m_{e}}^{\mathrm{OB}} h_{\mathrm{C}}\right)}{\sin ^{2}\left(k_{z m_{e}}^{\mathrm{OT}} h_{\mathrm{I}}\right)} \\
& \cdot \int_{h_{\mathrm{C}}}^{h_{\mathrm{O}}} \sin ^{2}\left(k_{z m_{h}}^{\mathrm{OT}}\left[h_{\mathrm{O}}-z\right]\right) d z \\
= & -4 \mu_{d}^{\mathrm{OB}}\left\{I_{1 h}+\frac{\mu_{d}^{\mathrm{OB}}}{\mu_{d}^{\mathrm{OT}}} \frac{\sin ^{2}\left(k_{z m_{h}}^{\mathrm{OB}} h_{\mathrm{C}}\right)}{\sin ^{2}\left(k_{z m_{h}}^{\mathrm{OT}} h_{\mathrm{I}}\right)} I_{2 h}\right\}
\end{aligned}
$$

where [11]

$$
\begin{aligned}
I_{1 h} & =\int_{0}^{h_{\mathrm{C}}} \sin ^{2}\left(k_{z m_{h}}^{\mathrm{OB}} z\right) d z \\
& =\frac{1}{k_{z m_{h}}^{\mathrm{OB}}}\left[-\frac{1}{4} \sin \left(2 k_{z m_{h}}^{\mathrm{OB}} h_{\mathrm{C}}\right)+\frac{k_{z m_{h}}^{\mathrm{OB}} h_{\mathrm{C}}}{2}\right] \\
I_{2 h} & =\int_{h_{\mathrm{O}}}^{h_{\mathrm{O}}} \sin ^{2}\left(k_{z m_{h}}^{\mathrm{OT}}\left[h_{\mathrm{O}}-z\right]\right) d z \\
& =\frac{1}{k_{z m_{h}}^{\mathrm{OT}}}\left[-\frac{1}{4} \sin \left(2 k_{z m_{h}}^{\mathrm{OT}} h_{\mathrm{I}}\right)+\frac{k_{z m_{h}}^{\mathrm{OT}} h_{\mathrm{I}}}{2}\right] .
\end{aligned}
$$




\section{IMPLICIT DYADIC GREEN'S FUNCTION CONSTRUCTION}

\section{A. Fields for Puck, Interior, and External Zone Interfaces}

Electromagnetic fields to be used in following continuity conditions employing the mode-matching method are summarized here from the previous section in the $\mathrm{O}$ zone, plus those found also in the $\mathrm{I}$ and $\mathrm{C}$ regions:

$$
\begin{aligned}
& E_{z}^{\mathrm{I}}(r, \phi, z) \\
& \quad=\sum_{m=0}^{\infty} \sum_{n=-\infty}^{\infty} A_{n m}^{e \mathrm{I}} \cos \left(k_{z m}^{\mathrm{I}}\left[z-h_{\mathrm{C}}\right] J_{n}\left(\sigma_{m}^{\mathrm{I}} r\right) e^{i n \phi}\right. \\
& H_{z}^{\mathrm{I}}(r, \phi, z) \\
& \quad=\sum_{m=1}^{\infty} \sum_{n=-\infty}^{\infty} A_{n m}^{h \mathrm{I}} \sin \left(k_{z m}^{\mathrm{I}}\left[z-h_{\mathrm{C}}\right]\right) K_{n}\left(\sigma_{m}^{\mathrm{I}} r\right) e^{i n \phi} \\
& E_{\phi}^{\mathrm{I}}(r, \phi, z) \\
& \quad=\sum_{m=1}^{\infty} \sum_{n=-\infty}^{\infty} i \sin \left(k_{z m}^{\mathrm{I}}\left[z-h_{\mathrm{C}}\right]\right) \\
& \quad \cdot\left[-s_{m}^{\mathrm{I}} A_{n m}^{h \mathrm{I}} \sigma_{m}^{\mathrm{I}} J_{n}^{\prime}\left(\sigma_{m}^{\mathrm{I}} r\right)+\frac{i p_{j}^{\mathrm{I}} n}{R} A_{n m}^{e \mathrm{I}} J_{n}\left(\sigma_{m}^{\mathrm{I}} r\right)\right] e^{i n \phi}
\end{aligned}
$$$$
H_{\phi}^{\mathrm{I}}(r, \phi, z)
$$$$
=\sum_{m=0}^{\infty} \sum_{n=-\infty}^{\infty} \cos \left(k_{z m}^{\mathrm{I}}\left[z-h_{\mathrm{C}}\right]\right)
$$$$
\cdot\left[\frac{i n p_{m}^{\mathrm{I}}}{R} A_{n m}^{h \mathrm{I}} J_{n}\left(\sigma_{m}^{\mathrm{I}} r\right)-u_{m}^{\mathrm{I}} A_{n m}^{e \mathrm{I}} \sigma_{m}^{\mathrm{I}} J_{n}^{\prime}\left(\sigma_{m}^{\mathrm{I}} r\right)\right] e^{i n \phi}
$$$$
E_{z}^{\mathrm{O}}(r, \phi, z)
$$$$
=\sum_{m_{e}=1}^{\infty} \sum_{n=-\infty}^{\infty} A_{n m_{e}}^{e \mathrm{OT}-} K_{n}\left(\sigma_{m_{e}}^{\mathrm{O}} r\right) \overline{\bar{Z}}_{m_{e}}^{e}(z) e^{i n \phi}
$$$$
H_{z}^{\mathrm{O}}(r, \phi, z)
$$$$
=\sum_{m_{h}=1}^{\infty} \sum_{n=-\infty}^{\infty} A_{n m_{h}}^{h \mathrm{OT}-} K_{n}\left(\sigma_{m_{h}}^{\mathrm{O}} r\right) \overline{\bar{Z}}_{m_{h}}^{h}(z) e^{i n \phi}
$$$$
E_{\phi}^{\mathrm{O}}(r, \phi, z)
$$$$
=\sum_{m_{h}=1}^{\infty} \sum_{n=-\infty}^{\infty}-s_{m_{h}}^{\mathrm{O}} A_{n m_{h}}^{h \mathrm{OT}}-\sigma_{m_{h}}^{\mathrm{O}} K_{n}^{\prime}\left(\sigma_{m_{h}}^{\mathrm{O}} r\right) \overline{\bar{Z}}_{m_{h_{l}}}^{h}(z)
$$$$
\cdot e^{i n \phi}+\sum_{m_{e}=1}^{\infty} \sum_{n=-\infty}^{\infty} \frac{i p_{m_{e}}^{\mathrm{O}} n}{r} A_{n m_{h}}^{e \mathrm{OT}}-K_{n}\left(\sigma_{m_{e}}^{\mathrm{O}} r\right) \frac{1}{i k_{z m_{e}}^{\mathrm{O}}}
$$$$
\cdot \frac{d \overline{\bar{Z}}_{m_{e}}^{e}(z)}{d z} e^{i n \phi}
$$$$
H_{\phi}^{\mathrm{O}}(r, \phi, z)
$$$$
=\sum_{m_{h}=1}^{\infty} \sum_{n=-\infty}^{\infty} \frac{i p_{m_{h}}^{\mathrm{O}} n}{r} A_{n m_{h}}^{h \mathrm{OT}-} K_{n}\left(\sigma_{m_{h}}^{\mathrm{O}} r\right) \frac{1}{i k_{z m_{h}}^{\mathrm{O}}}
$$$$
\cdot \frac{d \overline{\bar{Z}}_{m_{h}}^{h}(z)}{d z} e^{i n \phi}+\sum_{m_{e}=1}^{\infty} \sum_{n=-\infty}^{\infty}-u_{m_{e}}^{\mathrm{O}} A_{n m_{e}}^{e \mathrm{OT}}-\sigma_{m_{e}}^{\mathrm{O}}
$$$$
\cdot K_{n}^{\prime}\left(\sigma_{m_{e}}^{\mathrm{O}} r\right) \overline{\bar{Z}}_{m_{e}}^{e}(z) e^{i n \phi}
$$

$$
\begin{aligned}
E_{z}^{\mathrm{C}}(r, \phi, z) & \sum_{m=0}^{\infty} \sum_{n=-\infty}^{\infty} \cos \left(k_{z m}^{\mathrm{C}} z\right) \\
= & \cdot\left[{ }_{z}^{e} A_{n m N}^{1}(r) a_{n m 0}^{1}+{ }_{z}^{e} A_{n m N}^{2}(r) a_{n m 0}^{2}\right] e^{i n \phi} \\
E_{\phi}^{\mathrm{C}}(r, \phi, z) & \\
= & \sum_{m=1}^{\infty} \sum_{n=-\infty}^{\infty} i \sin \left(k_{z m}^{\mathrm{C}} z\right) \\
& \cdot\left[{ }_{\phi}^{e} A_{n m N}^{1}(r) a_{n m 0}^{1}+{ }_{\phi}^{e} A_{n m N}^{2}(r) a_{n m 0}^{2}\right] e^{i n \phi} \\
H_{z}^{\mathrm{C}}(r, \phi, z) & \sum_{m=1}^{\infty} \sum_{n=-\infty}^{\infty} i \sin \left(k_{z m}^{\mathrm{C}} z\right) \\
& \cdot\left[{ }_{z}^{h} A_{n m N}^{1}(r) a_{n m 0}^{1}+{ }_{z}^{h} A_{n m N}^{2}(r) a_{n m 0}^{2}\right] e^{i n \phi} \\
H_{\phi}^{\mathrm{C}}(r, \phi, z) & \\
= & \sum_{m=0}^{\infty} \sum_{n=-\infty}^{\infty} \cos \left(k_{z m}^{\mathrm{C}} z\right) \\
& \cdot\left[{ }_{\phi}^{h} A_{n m N}^{1}(r) a_{n m 0}^{1}+{ }_{\phi}^{h} A_{n m N}^{2}(r) a_{n m 0}^{2}\right] e^{i n \phi} .
\end{aligned}
$$

Field expressions in (130)-(133) for the circulator puck region are available in [2] and [10].

Use of tangential-field continuity at the $r=R$ interface will be used to connect the various regions, with $z$ the variable, i.e.,

$$
\left.\left\{E_{z}, E_{\phi}, H_{z}, H_{\phi}\right\}\right|_{r=R^{-}}=\left.\left\{E_{z}, E_{\phi}, H_{z}, H_{\phi}\right\}\right|_{r=R^{+}} \text {. }
$$

This expression must be applied at the $\mathrm{CO}$ and $\mathrm{IO}$ interfaces. It will not hold at the $\mathrm{CO}$ interface for the magnetic-field components because the $r=R$ surface contains singular forcing functions (delta functions). In the spirit of applying rigorous mode-matching theory by projecting testing functions on the continuity equations and integrating, we find for the IO interface

$$
\begin{aligned}
\int_{h_{\mathrm{C}}}^{h_{\mathrm{O}}} \psi_{e z m}^{\mathrm{I}} E_{z}^{\mathrm{I}} d z & =\int_{h_{\mathrm{C}}}^{h_{\mathrm{O}}} \psi_{e z m}^{\mathrm{I}} E_{z}^{\mathrm{O}} d z \\
\int_{h_{\mathrm{O}}}^{h_{\mathrm{O}}} \psi_{e \phi m}^{\mathrm{I}} E_{\phi}^{\mathrm{I}} d z & =\int_{h_{\mathrm{O}}}^{h_{\mathrm{O}}} \psi_{e \phi m}^{\mathrm{I}} E_{\phi}^{\mathrm{O}} d z \\
\int_{h_{\mathrm{O}}}^{h_{\mathrm{O}}} \psi_{h z m}^{\mathrm{I}} H_{z}^{\mathrm{I}} d z & =\int_{h_{\mathrm{O}}}^{h_{\mathrm{O}}} \psi_{h z m}^{\mathrm{I}} H_{z}^{\mathrm{O}} d z \\
\int_{h_{\mathrm{O}}}^{h_{\mathrm{O}}} \psi_{h \phi m}^{\mathrm{I}} H_{\phi}^{\mathrm{I}} d z & =\int_{h_{\mathrm{C}}}^{h_{\mathrm{O}}} \psi_{h \phi m}^{\mathrm{I}} H_{\phi}^{\mathrm{O}} d z
\end{aligned}
$$

and for the $\mathrm{CO}$ interface

$$
\begin{aligned}
& \int_{0}^{h_{\mathrm{C}}} \psi_{e, z m}^{\mathrm{C}} E_{z}^{\mathrm{C}} d z=\int_{0}^{h_{\mathrm{C}}} \psi_{e z m}^{\mathrm{C}} E_{z}^{\mathrm{O}} d z \\
& \int_{0}^{h_{\mathrm{C}}} \psi_{e \phi m}^{\mathrm{C}} E_{\phi}^{\mathrm{C}} d z=\int_{0}^{h_{\mathrm{C}}} \psi_{e \phi m}^{\mathrm{C}} E_{\phi}^{\mathrm{O}} d z .
\end{aligned}
$$

Lastly, for the $\mathrm{O}(\mathrm{C}+\mathrm{I})$ interface

$$
\begin{aligned}
& \int_{0}^{h_{\mathrm{O}}} \psi_{e z m}^{\mathrm{O}} E_{z}^{\mathrm{O}} d z=\int_{0}^{h_{\mathrm{C}}} \psi_{e z m}^{\mathrm{O}} E_{z}^{\mathrm{C}} d z+\int_{h_{\mathrm{C}}}^{h_{\mathrm{O}}} \psi_{e z m}^{\mathrm{O}} E_{z}^{\mathrm{I}} d z \\
& \int_{0}^{h_{\mathrm{O}}} \psi_{e \phi m}^{\mathrm{O}} E_{\phi}^{\mathrm{O}} d z=\int_{0}^{h_{\mathrm{C}}} \psi_{e \phi m}^{\mathrm{O}} E_{\phi}^{\mathrm{C}} d z+\int_{h_{\mathrm{C}}}^{h^{\mathrm{O}}} \psi_{e \phi m}^{\mathrm{O}} E_{\phi}^{\mathrm{I}} d z
\end{aligned}
$$


Here, $\psi_{\text {fjm }}^{i}$ are $z$-dependent testing functions of the particular region $i=\mathrm{I}, \mathrm{O}, \mathrm{C}$ as applied to the $f$-type of field-continuity equation for component $j$-type, with the mode index $m$. Projection of these functions on the field-continuity equations in the fashion of (135)-(142) allows their orthogonal properties to be used, with proper attention paid to weighting functions, thereby reducing the complexity of the eventual system of equations describing the problem. Wherever orthogonality is exploited, matrix sparsity is enhanced. For the exterior zone problem, the work required to determine the orthogonal properties of the scalar generating potentials is balanced by their convenience in use and sparse matrix behavior. We will use such potential properties here, noting that it is possible to use an external unloaded zone cavity testing function (with no orthogonal features, but easy to identify). These testing functions are

$$
\begin{aligned}
& \psi_{e z m}^{\mathrm{I}}=\psi_{h \phi m}^{\mathrm{I}}=\cos \left(k_{z m}^{\mathrm{I}}\left[z-h_{\mathrm{C}}\right]\right), \\
& k_{z m}^{\mathrm{I}}=\frac{m \pi}{h_{\mathrm{I}}} \quad m=0,1, \cdots, M_{\mathrm{I}}^{e, h} \\
& \psi_{h z m}^{\mathrm{I}}=\psi_{e \phi m}^{\mathrm{I}}=\sin \left(k_{z m}^{\mathrm{I}}\left[z-h_{\mathrm{C}}\right]\right), \\
& k_{z m}^{\mathrm{I}}=\frac{m \pi}{h_{\mathrm{I}}} \quad m=1,2, \cdots, M_{\mathrm{I}}^{h, e} \quad \\
& \psi_{e z m}^{\mathrm{C}}=\cos \left(k_{z m}^{\mathrm{C}} z\right), \quad k_{z m}^{\mathrm{C}}=\frac{m \pi}{h_{\mathrm{C}}} \quad m=0,1, \cdots, M_{\mathrm{C}} \\
& \psi_{e \phi m}^{\mathrm{C}}=\sin \left(k_{z m}^{\mathrm{C}} z\right), \quad k_{z m}^{\mathrm{C}}=\frac{m \pi}{h_{\mathrm{C}}} \quad m=1,2, \cdots, M_{\mathrm{C}} \\
& \psi_{e z m}^{\mathrm{O}}=\bar{Z}_{m_{e}}^{e}(z), \quad m=1,2, \cdots, M_{\mathrm{O}}^{e} \\
& \psi_{e \phi m}^{\mathrm{O}}=\bar{Z}_{m_{h}}^{h}(z), \quad m=1,2, \cdots, M_{\mathrm{O}}^{h} .
\end{aligned}
$$

Notice that the infinite summations contained in the field representations have been truncated to $M_{i}, i=\mathrm{I}, \mathrm{O}, \mathrm{C}$ in the respective regions, with mode type differentiation in the external zones. $M_{i}$ must be chosen with some care. $M_{\mathrm{I}}$ and $M_{\mathrm{C}}$ may be chosen relative to $M_{\mathrm{O}}$ (ignoring for the moment, mode-type differentiation) as

$$
M_{\mathrm{I}}=\frac{h_{\mathrm{I}}}{h_{\mathrm{O}}} M_{\mathrm{O}} \quad M_{\mathrm{C}}=\frac{h_{\mathrm{C}}}{h_{\mathrm{O}}} M_{\mathrm{O}}
$$

There is some flexibility in the specific ratio converting $M_{\mathrm{O}}$ into the other summation limits [12]. These issues are referred to as the relative convergence behavior. How large to choose $M_{\mathrm{O}}$ is an issue of absolute convergence, specifically how accurate an answer we desire for the problem solution.

\section{B. Nonsource-Governing Equations}

Selection of the subset (if a full set is not needed) of continuity equations is not unique [13], the choice being dependent upon individual inclination, sometimes numerical advantage, and the requirement that the number of equations equal the number of unknowns when the missing source equations are included (to be addressed later). Thus we choose (135), (136), (141), and (142), which, once the testing function projections and integrations are performed, become

$$
\begin{aligned}
& J_{n}\left(\sigma_{m}^{\mathrm{I}} R\right) A_{n m}^{e \mathrm{I}} \\
& =\frac{2 \varepsilon_{m}}{h_{\mathrm{I}}} \sum_{m_{e}=1}^{M_{\mathrm{Q}}^{e}} K_{n}\left(\sigma_{m_{e}}^{\mathrm{O}} R\right) R_{m_{e}}^{\mathrm{BT}-} A_{n m_{e}}^{e \mathrm{OT}-} I_{c Z m m_{e}}^{\mathrm{IO}}, \\
& m=0,1, \cdots, M_{\mathrm{I}}^{e} \\
& -s_{m}^{\mathrm{I}} \sigma_{m}^{\mathrm{I}} J_{n}^{\prime}\left(\sigma_{m}^{\mathrm{I}} R\right) A_{n m}^{h \mathrm{I}}+\frac{i p_{m}^{\mathrm{I}} n}{R} J_{n}\left(\sigma_{m}^{\mathrm{I}} R\right) A_{n m}^{e \mathrm{I}} \\
& =\frac{2}{i h_{\mathrm{I}}} \sum_{m_{h}=1}^{M_{\mathrm{O}}^{h_{h}}}-s_{m_{h}}^{\mathrm{O}} \sigma_{m_{h}}^{\mathrm{O}} K_{n}^{\prime}\left(\sigma_{m_{h}}^{\mathrm{O}} R\right) R_{m_{h}}^{\mathrm{BT}-} A_{n m_{h_{h}}}^{h \mathrm{OT}-} \\
& \cdot I_{s Z m m_{h}}^{\mathrm{IO}+}+\frac{2}{i h_{\mathrm{I}}} \sum_{m_{e}=1}^{M_{\mathrm{O}}^{e}} \frac{i p_{m_{e}}^{\mathrm{O}} n}{R} K_{n}\left(\sigma_{m_{e}}^{\mathrm{O}} R\right) R_{m_{e}}^{\mathrm{BT}-} \\
& \text { - } A_{n m_{e}}^{e \mathrm{OT}-} I_{s Z m m_{e}}^{\mathrm{IO}-}, \quad m=1,2, \cdots, M_{\mathrm{I}}^{h} \\
& K_{n}\left(\sigma_{m_{e}}^{\mathrm{O}} R\right)_{m_{e}}^{\mathrm{BT}-} C_{m_{e}}^{e} A_{n m_{e}}^{e \mathrm{OT}-} \\
& =\varepsilon_{d}^{\mathrm{OB}} \sum_{m=0}^{M_{\mathrm{C}}}\left[{ }_{z}^{e} A_{n m}^{1} a_{n m 0}^{1}+{ }_{z}^{e} A_{n m}^{2} A_{n m 0}^{2}\right] I_{Z c m_{e} m}^{\mathrm{OC}} \\
& +\varepsilon_{d}^{\mathrm{OT}} \sum_{m=0}^{M_{\mathrm{I}}^{e}} J_{n}\left(\sigma_{m}^{\mathrm{I}} R\right) A_{n m}^{e \mathrm{I}} I_{Z c m_{e} m}^{\mathrm{OI}}, \\
& m_{e}=1,2, \cdots, M_{\mathrm{O}}^{e} \\
& -\bar{s}_{m_{h}}^{\mathrm{O}} \sigma_{m_{h}}^{\mathrm{O}} K_{n}^{\prime}\left(\sigma_{m_{h}}^{\mathrm{O}} R\right) R_{m_{h}}^{\mathrm{BT}-} C_{m_{h}}^{h} A_{n m_{h}}^{h \mathrm{OT}-} \\
& +\sum_{m_{e}=1}^{M_{\mathrm{Q}}^{\epsilon}} \frac{i n}{R} K_{n}\left(\sigma_{m_{e}}^{\mathrm{O}} R\right) R_{m_{h}}^{\mathrm{BT}-} A_{n m_{e}}^{e \mathrm{OT}-} I_{Z Z m_{h} m_{e}}^{\mathrm{OO}} \\
& \left.=\sum_{m=1}^{M_{\mathrm{C}}}{ }_{\phi}^{e} A_{n m N}^{1}(R) a_{n m^{\prime} 0}^{1}+{ }_{\phi}^{e} A_{n m N}^{2}(R) a_{n m 0}^{2}\right] \\
& \cdot I_{Z s m_{h} m}^{\mathrm{OC}}+\sum_{m=1}^{M_{\mathrm{I}}^{h_{h}}}-i s_{m}^{\mathrm{I}} \sigma_{m}^{\mathrm{I}} J_{n}^{\prime}\left(\sigma_{m}^{\mathrm{I}} R\right) A_{n m}^{h \mathrm{I}} I_{Z s m_{h} m}^{\mathrm{OI}+} \\
& +\sum_{m=1}^{M_{\mathrm{I}}^{e}} i \frac{i p_{m}^{\mathrm{I}} n}{R} J_{n}\left(\sigma_{m}^{\mathrm{I}} R\right) A_{n m}^{e \mathrm{I}} I_{Z s m_{h} m}^{\mathrm{OI}+}, \\
& m_{h}=1,2, \cdots, M_{\mathrm{O}}^{h} .
\end{aligned}
$$

In these previous relationships, for the internal I region

$$
\left(k_{d}^{\mathrm{I}}\right)^{2}=\left(\sigma_{m}^{\mathrm{I}}\right)^{2}+\left(k_{z m}^{\mathrm{I}}\right)^{2} .
$$

Also, the normalized $s_{m_{h}}^{\mathrm{O}}$ constant used in (153) is

$$
\bar{s}_{m_{h}}^{\mathrm{O}}=\frac{s_{m_{h}}^{\mathrm{O}}}{\mu(z)} .
$$

The overlap integrals found in (150)-(153) are given by (156)-(165), shown on the following page [11].

Orthogonality relationships relevant to these equations are found in (104) and (116) for, respectively, $\bar{Z}_{m_{e}}^{e}$ and $\bar{Z}_{m_{h}}^{h}$ [used 


$$
\begin{aligned}
& I_{c Z m m_{e}}^{\mathrm{IO}}=\int_{h_{\mathrm{C}}}^{h_{\mathrm{O}}} \cos \left(\frac{m \pi}{h_{\mathrm{I}}}\left[z-h_{\mathrm{C}}\right]\right) \bar{Z}_{m_{e}}^{e}(z) d z \\
& =2 \frac{\varepsilon_{d}^{\mathrm{OB}}}{\varepsilon_{d}^{\mathrm{OT}}} \frac{\cos \left(k_{z m_{e}}^{\mathrm{OB}} h_{\mathrm{C}}\right)}{\cos \left(k_{z m_{e}}^{\mathrm{OT}} h_{\mathrm{I}}\right)} \int_{h_{\mathrm{C}}}^{h_{\mathrm{O}}} \cos \left(\frac{m \pi}{h_{\mathrm{I}}}\left[z-h_{\mathrm{C}}\right]\right) \cos \left(k_{z m_{e}}^{\mathrm{OT}}\left[z-h_{\mathrm{O}}\right]\right) d z \\
& =2 \frac{\varepsilon_{d}^{\mathrm{OB}}}{\varepsilon_{d}^{\mathrm{OT}}} \frac{\cos \left(k_{z m_{e}}^{\mathrm{OB}} h_{\mathrm{C}}\right)}{\cos \left(k_{z m_{e}}^{\mathrm{OT}} h_{\mathrm{I}}\right)} \frac{k_{z m_{e}}^{\mathrm{OT}}}{\left(k_{z m_{e}}^{\mathrm{OT}}\right)^{2}-\left(m \pi / h_{\mathrm{I}}\right)^{2}} \sin \left(k_{z m_{e}}^{\mathrm{OT}} h_{\mathrm{I}}\right) \\
& I_{s Z m m_{h}}^{\mathrm{IO}+}=\int_{h_{\mathrm{C}}}^{h_{\mathrm{O}}} \sin \left(\frac{m \pi}{h_{\mathrm{I}}}\left[z-h_{\mathrm{C}}\right]\right) \bar{Z}_{m_{h}}^{h}(z) d z \\
& =-2 i \frac{\mu_{d}^{\mathrm{OB}}}{\mu_{d}^{\mathrm{OT}}} \frac{\sin \left(k_{z m_{h}}^{\mathrm{OB}} h_{\mathrm{C}}\right)}{\sin \left(k_{z m_{h}}^{\mathrm{OT}} h_{\mathrm{I}}\right)} \int_{h_{\mathrm{C}}}^{h_{\circ}} \sin \left(\frac{m \pi}{h_{\mathrm{I}}}\left[z-h_{\mathrm{C}}\right]\right) \sin \left(k_{z m_{h}}^{\mathrm{OT}}\left[z-h_{\mathrm{O}}\right]\right) d z \\
& =-2 i \frac{\mu_{d}^{\mathrm{OB}}}{\mu_{d}^{\mathrm{OT}}} \frac{\sin \left(k_{z m_{h}}^{\mathrm{OB}} h_{\mathrm{C}}\right)}{\sin \left(k_{z m_{h}}^{\mathrm{OT}} h_{\mathrm{I}}\right)} \frac{m \pi / h_{\mathrm{I}}}{\left(k_{z m_{h}}^{\mathrm{OT}}\right)^{2}-\left(m \pi / h_{\mathrm{I}}\right)^{2}} \sin \left(k_{z m_{h}}^{\mathrm{OT}} h_{\mathrm{I}}\right) \\
& I_{s Z m m_{e}}^{\mathrm{IO}-}=\int_{h_{\mathrm{C}}}^{h_{\mathrm{O}}} \sin \left(\frac{m \pi}{h_{\mathrm{I}}}\left[z-h_{\mathrm{C}}\right]\right) \frac{1}{i k_{z m_{e}}^{\mathrm{OT}}} \frac{d \bar{Z}_{m_{e}}^{e}(z)}{d z} d z \\
& =\int_{h_{\mathrm{C}}}^{h_{\mathrm{O}}} \sin \left(\frac{m \pi}{h_{\mathrm{I}}}\left[z-h_{\mathrm{C}}\right]\right) \bar{Z}_{m_{e}}^{e-}(z) d z \\
& =2 i \frac{\varepsilon_{d}^{\mathrm{OB}}}{\varepsilon_{d}^{\mathrm{OT}}} \frac{\cos \left(k_{z m_{e}}^{\mathrm{OB}} h_{\mathrm{C}}\right)}{\cos \left(k_{z m_{e}}^{\mathrm{OT}} h_{\mathrm{I}}\right)} \int_{h_{\mathrm{C}}}^{h_{\mathrm{O}}} \sin \left(\frac{m \pi}{h_{\mathrm{I}}}\left[z-h_{\mathrm{C}}\right]\right) \sin \left(k_{z m_{e}}^{\mathrm{OT}}\left[z-h_{\mathrm{O}}\right]\right) d z \\
& =2 i \frac{\varepsilon_{d}^{\mathrm{OB}}}{\varepsilon_{d}^{\mathrm{OT}}} \frac{\cos \left(k_{z m_{e}}^{\mathrm{OB}} h_{\mathrm{C}}\right)}{\cos \left(k_{z m_{e}}^{\mathrm{OT}} h_{\mathrm{I}}\right)} \frac{m \pi / h_{\mathrm{I}}}{\left(k_{z m_{e}}^{\mathrm{OT}}\right)^{2}-\left(m \pi / h_{\mathrm{I}}\right)^{2}} \sin \left(k_{z m_{e}}^{\mathrm{OT}} h_{\mathrm{I}}\right) \\
& I_{Z c m_{e} m}^{\mathrm{OC}}=\int_{0}^{h_{\mathrm{C}}} \bar{Z}_{m_{e}}^{e}(z) \cos \left(k_{z m}^{\mathrm{C}} z\right) d z=\int_{0}^{h_{\mathrm{C}}} 2 \cos \left(k_{z m_{e}}^{\mathrm{OB}} z\right) \cos \left(k_{z m}^{\mathrm{C}} z\right) d z=2(-1)^{m} \frac{k_{z m_{e}}^{\mathrm{OB}}}{\left(k_{z m_{e}}^{\mathrm{OB}}\right)^{2}-\left(k_{z m}^{\mathrm{C}}\right)^{2}} \sin \left(k_{z m_{e}}^{\mathrm{OB}} h_{\mathrm{C}}\right) \\
& I_{Z c m_{e} m}^{\mathrm{OI}}=\int_{h_{\mathrm{C}}}^{h_{\mathrm{O}}} \bar{Z}_{m_{e}}^{e}(z) \cos \left(\frac{m \pi}{h_{\mathrm{I}}}\left[z-h_{\mathrm{C}}\right]\right) d z=I_{c Z m m_{e}}^{\mathrm{IO}} \\
& I_{Z Z m_{h} m_{e}}^{\mathrm{OO}}=\int_{0}^{h_{\mathrm{O}}} p_{m_{e}}^{\mathrm{O}}(z) \bar{Z}_{m_{h}}^{h}(z) \bar{Z}_{m_{e}}^{e-}(z) d z \\
& =p_{m_{e}}^{\mathrm{OB}} \int_{0}^{h_{\mathrm{C}}} \bar{Z}_{m_{h}}^{h \mathrm{~B}}(z) \bar{Z}_{m_{e}}^{e \mathrm{~B}-}(z) d z+p_{m_{e}}^{\mathrm{OT}} \int_{h_{C}}^{h_{\circ}} \bar{Z}_{m_{h}}^{h \mathrm{~T}}(z) \bar{Z}_{m_{e}}^{e \mathrm{~T}-}(z) d z \\
& =p_{m_{e}}^{\mathrm{OB}} I_{s s m_{h} m_{e}}^{\mathrm{BB}}+p_{m_{e}}^{\mathrm{OT}} I_{s s m_{h}}^{\mathrm{TT}} m_{e} \\
& I_{s s m_{h} m_{e}}^{\mathrm{BB}}=-4 \int_{0}^{h_{\mathrm{C}}} \sin \left(k_{z m_{h}}^{\mathrm{OB}} z\right) \sin \left(k_{z m_{e}}^{\mathrm{OB}} z\right) d z \\
& =\frac{4}{\left(k_{z m_{h}}^{\mathrm{OB}}\right)^{2}-\left(k_{z m_{e}}^{\mathrm{OB}}\right)^{2}}\left[k_{z m_{h}}^{\mathrm{OB}} \cos \left(k_{z m_{h}}^{\mathrm{OB}} h_{\mathrm{C}}\right) \sin \left(k_{z m_{e}}^{\mathrm{OB}} h_{\mathrm{C}}\right)-k_{z m_{e}}^{\mathrm{OB}} \sin \left(k_{z m_{h}}^{\mathrm{OB}} h_{\mathrm{C}}\right) \cos \left(k_{z m_{e}}^{\mathrm{OB}} h_{\mathrm{C}}\right)\right] \\
& I_{s s m_{h} m_{e}}^{\mathrm{TT}}=4 \frac{\mu_{d}^{\mathrm{OB}}}{\mu_{d}^{\mathrm{OT}}} \frac{\varepsilon_{d}^{\mathrm{OB}}}{\varepsilon_{d}^{\mathrm{OT}}} \frac{\sin \left(k_{z m_{h}}^{\mathrm{OB}} h_{\mathrm{C}}\right)}{\sin \left(k_{z m_{h}}^{\mathrm{OT}} h_{\mathrm{I}}\right)} \frac{\cos \left(k_{z m_{e}}^{\mathrm{OB}} h_{\mathrm{C}}\right)}{\cos \left(k_{z m_{e}}^{\mathrm{OT}} h_{\mathrm{I}}\right)} \int_{h_{\mathrm{C}}}^{h_{\mathrm{O}}} \sin \left(k_{z m_{h}}^{\mathrm{OT}}\left[z-h_{\mathrm{O}}\right]\right) \sin \left(k_{z m_{e}}^{\mathrm{OT}}\left[z-h_{\mathrm{O}}\right]\right) d z \\
& =4 \frac{\mu_{d}^{\mathrm{OB}}}{\mu_{d}^{\mathrm{OT}}} \frac{\varepsilon_{d}^{\mathrm{OB}}}{\varepsilon_{d}^{\mathrm{OT}}} \frac{\sin \left(k_{z m_{h}}^{\mathrm{OB}} h_{\mathrm{C}}\right)}{\sin \left(k_{z m_{h}}^{\mathrm{OT}} h_{\mathrm{I}}\right)} \frac{\cos \left(k_{z m_{e}}^{\mathrm{OB}} h_{\mathrm{C}}\right)}{\cos \left(k_{z m_{e}}^{\mathrm{OT}} h_{\mathrm{I}}\right)} \frac{k_{z m_{e}}^{\mathrm{OB}} \sin \left(k_{z m_{h}}^{\mathrm{OB}} h_{\mathrm{C}}\right) \cos \left(k_{z m_{e}}^{\mathrm{OB}} h_{\mathrm{C}}\right)-k_{z m_{h}}^{\mathrm{OB}} \cos \left(k_{z m_{h}}^{\mathrm{OB}} h_{\mathrm{C}}\right) \sin \left(k_{z m_{e}}^{\mathrm{OB}} h_{\mathrm{C}}\right)}{\left(k_{z m_{h}}^{\mathrm{OB}}\right)^{2}-\left(k_{z m_{e}}^{\mathrm{OB}}\right)^{2}} \\
& I_{Z s m_{h} m}^{\mathrm{OC}}=\int_{0}^{h_{\mathrm{C}}} \bar{Z}_{m_{h}}^{h}(z) i \sin \left(k_{z m}^{\mathrm{C}} z\right) d z=-2 \int_{0}^{h_{\mathrm{C}}} \sin \left(k_{z m_{h}}^{\mathrm{OB}} z\right) \sin \left(k_{z m}^{\mathrm{C}} z\right) d z=\frac{2 k_{z m}^{\mathrm{C}}}{\left(k_{z m}^{\mathrm{C}}\right)^{2}-\left(k_{z m_{h}}^{\mathrm{OB}}\right)^{2}}(-1)^{m} \sin \left(k_{z m_{h}}^{\mathrm{OB}} h_{\mathrm{C}}\right) \\
& I_{Z s m_{h} m}^{\mathrm{OI}+}=\int_{h_{\mathrm{C}}}^{h_{\mathrm{O}}} \bar{Z}_{m_{h}}^{h}(z) \sin \left(\frac{m \pi}{h_{\mathrm{I}}}\left[z-h_{\mathrm{C}}\right]\right) d z=I_{s Z m m_{h}}^{\mathrm{IO}+}
\end{aligned}
$$


in (152) and (153)], and below for (150) and (151):

$$
\begin{aligned}
\int_{h_{\mathrm{C}}}^{h_{\mathrm{O}}} & \psi_{e z m}^{\mathrm{I}} \psi_{e z m^{\prime}}^{\mathrm{I}} d z \\
& =\int_{h_{\mathrm{C}}}^{h_{\mathrm{O}}} \cos \left(\frac{m \pi}{h_{\mathrm{I}}}[z-h]\right) \cos \left(\frac{m^{\prime} \pi}{h_{\mathrm{I}}}[z-h]\right) d z \\
& =\frac{h_{\mathrm{I}}}{2 \varepsilon_{m}} \delta_{m m^{\prime}} \\
\int_{h_{\mathrm{C}}}^{h_{\mathrm{O}}} \psi_{e \phi m}^{\mathrm{I}} \psi_{e \phi m^{\prime}}^{\mathrm{I}} d z & \int_{h_{\mathrm{O}}}^{h_{\mathrm{O}}} \sin \left(\frac{m \pi}{h_{\mathrm{I}}}[z-h]\right) \sin \left(\frac{m^{\prime} \pi}{h_{\mathrm{I}}}[z-h]\right) d z \\
= & \frac{h_{\mathrm{I}}}{2} \delta_{m m^{\prime}}, \quad m, m^{\prime}>0
\end{aligned}
$$

where

$$
\varepsilon_{m}= \begin{cases}1 / 2, & m=0 \\ 1, & m>0\end{cases}
$$

Also

$$
\bar{Z}_{m_{e}}^{e-}(z)=\frac{1}{i k_{z m_{e}}^{\mathrm{OT}}} \frac{d \bar{Z}_{m_{e}}^{e}(z)}{d z}
$$

was used in (158), and a similar formula exists for the " $h$ " mode.

Four-system equation-set (150)-(153) constitute $M_{\mathrm{I}}^{e}+M_{\mathrm{I}}^{h}+$ $M_{\mathrm{O}}^{e}+M_{\mathrm{O}}^{h}+1$ individual equations $\left(2\left(M_{\mathrm{I}}+M_{\mathrm{O}}\right)+1\right.$ if the " $e$ " and " $h$ " mode index limits are the same), but the unknowns are

$$
\begin{array}{cl}
a_{n m 0}^{1}, & m=0,1, \cdots, M_{\mathrm{C}} \\
a_{n m 0}^{2}, & m=1,2, \cdots, M_{\mathrm{C}} \\
a_{n m_{e}}^{e \mathrm{OT}-}, & m_{e}=1,2, \cdots, M_{\mathrm{O}}^{e} \\
A_{n m_{h}}^{h \mathrm{OT}-}, & m_{h}=1,2, \cdots, M_{\mathrm{O}}^{h} \\
A_{n m}^{e \mathrm{I}}, & m=0,1, \cdots, M_{\mathrm{I}}^{e} \\
A_{n m}^{h \mathrm{I}}, & m=1,2, \cdots, M_{\mathrm{I}}^{h}
\end{array}
$$

making a total of $M_{\mathrm{I}}^{e}+M_{\mathrm{I}}^{h}+M_{\mathrm{O}}^{e}+M_{\mathrm{O}}^{h}+2\left(M_{\mathrm{C}}+1\right)$ of them $\left(2\left(M_{\mathrm{I}}+M_{\mathrm{O}}+M_{\mathrm{C}}+1\right)\right.$ if the " $e$ " and " $h$ " mode index limits are the same). Notice that the $m=0$ case has been left off of the second radial-mode coefficient index listing (170b) because it corresponds to the zero perpendicular thickness situation and we expect the first radial mode to completely dominate. Therefore, we see that exactly $2 M_{\mathrm{C}}+1$ more equations are required to describe the structure.

\section{Source-Governing Equations}

The missing equations come from two source equations governing the exchange of energy between the magnetic delta forcing functions acting on the $r=R$ surface through a point aperture, and the puck structure. The components of the surface magnetic field are chosen to couple the external environment to the circulator puck structure, thereby defining a Green's function construction. The two source equations are

$$
H_{\phi A}(\phi, z)=H_{\phi^{\prime}}^{p t} \delta\left(z-z^{\prime}\right) \delta\left(\phi-\phi^{\prime}\right) \Delta \phi^{\prime}+H_{\phi}^{\mathrm{O}}(\phi, z)
$$

$$
H_{z A}(\phi, z)=H_{z^{\prime}}^{p t} \delta\left(z-z^{\prime}\right) \delta\left(\phi-\phi^{\prime}\right) \Delta \phi^{\prime}+H_{z}^{\mathrm{O}}(\phi, z) .
$$

Both $H_{\phi A}$ and $H_{z A}$ may be expressed by a Fourier series constructed from the same expansion functions as used to represent the circulator puck field components on the interval $\left(0, h_{\mathrm{C}}\right)$ by simply using the extended field on $\left(-h_{\mathrm{C}}, h_{\mathrm{C}}\right)$ [14] as follows:

$$
\begin{aligned}
H_{\phi A}(\phi, z)= & \sum_{m=0}^{\infty} H_{\phi A m}^{c} \cos \left(\frac{m \pi z}{h_{\mathrm{C}}}\right) \\
& +\sum_{m=1}^{\infty} H_{\phi A m}^{s} \sin \left(\frac{m \pi z}{h_{\mathrm{C}}}\right) \\
H_{z A}(\phi, z)= & \sum_{m=0}^{\infty} H_{z A m}^{c} \cos \left(\frac{m \pi z}{h_{\mathrm{C}}}\right) \\
& +\sum_{m=1}^{\infty} H_{z A m}^{s} \sin \left(\frac{m \pi z}{h_{\mathrm{C}}}\right) \\
H_{\phi A m}^{c}(\phi)= & \frac{\varepsilon_{m}}{h_{\mathrm{C}}} \int_{-h_{\mathrm{C}}}^{h_{\mathrm{C}}} H_{\phi A}^{e x}(\phi, z) \cos \left(\frac{m \pi z}{h_{\mathrm{C}}}\right) d z \\
= & \frac{2 \varepsilon_{m}}{h_{\mathrm{C}}} \int_{0}^{h_{\mathrm{C}}} H_{\phi A}(\phi, z) \cos \left(\frac{m \pi z}{h_{\mathrm{C}}}\right) d z \\
H_{z A m}^{s}(\phi)= & \frac{1}{h_{\mathrm{C}}} \int_{-h_{\mathrm{C}}}^{h_{\mathrm{C}}} H_{z A}^{e z}(\phi, z) \sin \left(\frac{m \pi z}{h_{\mathrm{C}}}\right) d z \\
= & \frac{2}{h_{\mathrm{C}}} \int_{0}^{h_{\mathrm{C}}} H_{z A}(\phi, z) \sin \left(\frac{m \pi z}{h_{\mathrm{C}}}\right) d z \\
H_{\phi A m}^{s}= & H_{\phi A m}^{c}=0 .
\end{aligned}
$$

Here "ex" indicates extended field and (177) results from the $\cos$ or $\sin$ nature of the field component variation within the circulator puck. It is convenient to retain the exponential Fourier series on $(-\pi, \pi)$ for an additional representation of the $\phi$-dependent coefficients found in the $z$-expansions (173) and (174). Thus

$$
\begin{aligned}
H_{\phi A m}^{c}(\phi) & =\sum_{n=-\infty}^{\infty} H_{\phi A m n}^{c} e^{i n \phi} \\
H_{z A m}^{s}(\phi) & =\sum_{n=-\infty}^{\infty} H_{z A m n}^{s} e^{i n \phi} \\
H_{\phi A m n}^{c} & =\frac{1}{2 \pi} \int_{-\pi}^{\pi} H_{\phi A m}^{c}(\phi) e^{-i n \phi} d \phi \\
H_{z A m n}^{s} & =\frac{1}{2 \pi} \int_{-\pi}^{\pi} H_{z A m}^{s}(\phi) e^{-i n \phi} d \phi .
\end{aligned}
$$

With the foregoing information, namely (171)-(181), the proper constraints on the sources, can be imposed. It is done through the requirements that the tangential magnetic-field components to the $r=R$ surface be continuous in a limiting process just to the inside and outside of the device perimeter

$$
\begin{gathered}
\left.H_{\phi}^{c}\right|_{r=R^{-}}=\left.H_{\phi A}\right|_{r=R^{+}} \\
\left.H_{z}^{c}\right|_{r=R^{-}}=\left.H_{z A}\right|_{r=R^{+}} .
\end{gathered}
$$

Use of the azimuthal orthogonality property and recognizing the similarity of the perpendicular coordinate expansions in 
both the puck and aperture (source) surface

$$
\begin{aligned}
H_{\phi m n}^{\mathrm{cir}} & =H_{\phi A m n}^{c} \\
H_{z m n}^{\mathrm{cir}} & =H_{z A m n}^{c}
\end{aligned}
$$

where we have written out cir to emphasize the difference between the puck expansion coefficients and the aperture $m n$th coefficient, which happens to be a cosine type. Placing (175) into (180) gives, considering first the LHS of (183a)

$$
\begin{aligned}
H_{\phi A m n}^{c}= & \frac{\varepsilon_{m}}{\pi h_{\mathrm{C}}} \int_{0}^{h_{\mathrm{C}}} \int_{-\pi}^{\pi} H_{\phi A}(\phi, z) \\
& \cdot \cos \left(\frac{m \pi z}{h_{\mathrm{C}}}\right) e^{-i n \phi} d \phi d z
\end{aligned}
$$

Next, insert the source equation (171) into (184) as follows:

$$
\begin{aligned}
H_{\phi A m n}^{c}= & \frac{\varepsilon_{m}}{\pi h_{\mathrm{C}}} \int_{0}^{h_{\mathrm{C}}} H_{\phi^{\prime}}^{p t} \delta\left(z-z^{\prime}\right) \cos \left(\frac{m \pi z}{h_{\mathrm{C}}}\right) d z \\
& \cdot \int_{-\pi}^{\pi} \delta\left(\phi-\phi^{\prime}\right) \Delta \phi^{\prime} e^{-i n \phi} d \phi d z \\
& +\frac{\varepsilon_{m}}{\pi h_{\mathrm{C}}} \int_{0}^{h_{\mathrm{C}}} \int_{-\pi}^{\pi} H_{\phi}^{\mathrm{O}}(\phi, z) \cos \left(\frac{m \pi z}{h_{\mathrm{C}}}\right) \\
& \cdot e^{-i n \phi} d \phi d z \\
= & \frac{\varepsilon_{m}}{\pi h_{\mathrm{C}}} \cos \left(\frac{m \pi z}{h_{\mathrm{C}}}\right) H_{\phi^{\prime}}^{p t} \Delta \phi^{\prime} e^{-i n \phi^{\prime}+“ \mathrm{O} \text { ” integral. }}
\end{aligned}
$$

Retrieving $H_{\phi}^{\mathrm{O}}$ in (129), the second integral in (185) can be evaluated as follows for the OB and OT regions being identical, demonstrating the reduction of one infinite summation:

"O" integral

$$
\begin{aligned}
=\frac{\varepsilon_{m}}{\pi h_{\mathrm{C}}} \sum_{m^{\prime}=0}^{\infty} \sum_{n^{\prime}=-\infty}^{\infty}\left[\frac{i n^{\prime} p_{m^{\prime}}^{\mathrm{O}}}{R} A_{n^{\prime} m^{\prime}}^{h \mathrm{O}} K_{n^{\prime}}\left(\sigma_{m^{\prime}}^{\mathrm{O}} R\right)\right. & \left.\quad-u_{m^{\prime}}^{\mathrm{O}} A_{n^{\prime} m^{\prime}}^{e \mathrm{O}} \sigma_{m^{\prime}}^{\mathrm{O}} K_{n^{\prime}}^{\prime}\left(\sigma_{m^{\prime}}^{\mathrm{O}} R\right)\right] \\
& \times \int_{-\pi}^{\pi} e^{i\left(n^{\prime}-n\right) \phi} d \phi \int_{0}^{h_{\mathrm{C}}} \cos \left(\frac{m^{\prime} \pi}{h_{\mathrm{O}}} z\right) \cos \left(\frac{m \pi}{h_{\mathrm{C}}}\right) d z \\
= & \frac{\varepsilon_{m}}{\pi h_{\mathrm{C}}} \sum_{m^{\prime}=0}^{\infty} \sum_{n^{\prime}=-\infty}^{\infty}\left[n^{\prime} m^{\prime}\right] 2 \pi \delta_{n^{\prime} n} I_{c m^{\prime} m}^{\mathrm{OC}} \\
= & \frac{2 \varepsilon_{m}}{h_{\mathrm{C}}} \sum_{m^{\prime}=0}^{\infty}\left[n m^{\prime}\right] I_{c m^{\prime} m^{\mathrm{OC}}} .
\end{aligned}
$$

At this stage, constraint (183a) can be imposed, invoking (133) for the RHS, using the previous (185) and (186) results. The fifth system equation, containing $M_{\mathrm{C}}+1$ subequations, can now be stated as

$$
\begin{aligned}
{ }_{\phi}^{h} A_{n m N}^{1}(R) a_{n m 0}^{1}+{ }_{\phi}^{h} A_{n m N}^{2}(R) a_{n m 0}^{2} \\
=\frac{\varepsilon_{m}}{\pi h_{\mathrm{C}}} \cos \left(\frac{m \pi z}{h_{\mathrm{C}}}\right) H_{\phi^{\prime}}^{p t} \Delta \phi^{\prime} e^{-i n \phi^{\prime}}+\frac{2 \varepsilon_{m}}{h_{\mathrm{C}}} \\
\cdot \sum_{m_{h}=1}^{M_{\mathrm{O}}^{h_{2}}} \frac{i p_{m_{h}}^{\mathrm{OB}} n}{R} K_{n}\left(\sigma_{m_{h}}^{\mathrm{O}} R\right) R_{m_{h}}^{\mathrm{BT}-} A_{n m_{h}}^{h \mathrm{OT}-}
\end{aligned}
$$

$$
\begin{aligned}
& \cdot I_{c Z m m_{h}}^{\mathrm{CO}}+\frac{2 \varepsilon_{m}}{h_{\mathrm{C}}} \sum_{m_{e}=1}^{M_{\mathrm{O}}^{e}}-u_{m_{e}}^{\mathrm{OB}} \sigma_{m_{e}}^{\mathrm{O}} K_{n}^{\prime}\left(\sigma_{m_{e}}^{\mathrm{O}} R\right) \\
& \cdot R_{m_{e}}^{\mathrm{BT}-} A_{n m_{e}}^{e \mathrm{OT}-} I_{c Z m m_{e}}^{\mathrm{CO}}, \quad m=0,1, \cdots, M_{\mathrm{C}} .
\end{aligned}
$$

Overlap integrals on the RHS of (187) are determined to be

$$
\begin{aligned}
I_{c Z m m_{h}}^{\mathrm{CO}} & =\int_{0}^{h_{\mathrm{C}}} \cos \left(k_{z m}^{\mathrm{C}} z\right)\left(1 / i k_{z m_{h}}^{\mathrm{OB}}\right)\left(d \bar{Z}_{m_{h}}(z) / d z\right) d z \\
& =2 \int_{0}^{h_{\mathrm{C}}} \cos \left(k_{z m}^{\mathrm{C}} z\right) \cos \left(k_{z m_{h}}^{\mathrm{OB}} z\right) d z \\
& =\frac{2 k_{z m_{h}}^{\mathrm{OB}}}{\left(k_{z m_{h}}^{\mathrm{OB}}\right)^{2}-\left(k_{z m}^{\mathrm{C}}\right)^{2}}(-1)^{m} \sin \left(k_{z m_{h}}^{\mathrm{OB}} h_{\mathrm{C}}\right) \\
I_{c Z m m_{e}}^{\mathrm{CO}} & =\int_{0}^{h_{\mathrm{C}}} \cos \left(k_{z m}^{\mathrm{C}} z\right) \bar{Z}_{m_{e}}^{e}(z) d z \\
& =2 \int_{0}^{h_{\mathrm{C}}} \cos \left(k_{z m}^{\mathrm{C}} z\right) \cos \left(k_{z m_{e}}^{\mathrm{OB}} z\right) d z \\
& =\frac{2 k_{z m_{e}}^{\mathrm{OB}}}{\left(k_{z m_{e}}^{\mathrm{OB}}\right)^{2}-\left(k_{z m}^{\mathrm{C}}\right)^{2}}(-1)^{m} \sin \left(k_{z m_{e}}^{\mathrm{OB}} h_{\mathrm{C}}\right) .
\end{aligned}
$$

The sixth system equation containing $M_{\mathrm{C}}$ subequations is found by treating (182b). Place (176) into (181), obtaining

$$
H_{z A m n}^{c}=\frac{1}{\pi h_{\mathrm{C}}} \int_{0}^{h_{\mathrm{C}}} \int_{-\pi}^{\pi} H_{z A}(\phi, z) \sin \left(\frac{m \pi z}{h_{\mathrm{C}}}\right) e^{-i n \phi} d \phi d z .
$$

Inserting the aperture source expression (172) for the perpendicular magnetic-field component into (190) gives

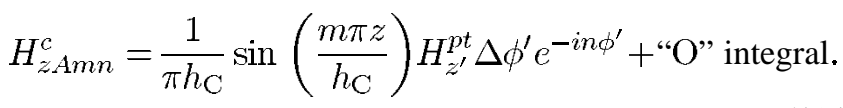

Using (127), the outside integral second term is expressed as

$$
\begin{aligned}
\text { "O” integral }= & \frac{1}{\pi h_{\mathrm{C}}} \sum_{m_{h}=1}^{\infty} \sum_{n^{\prime}=-\infty}^{\infty} A_{n^{\prime} m_{h}}^{h \mathrm{OB}-} K_{n^{\prime}}\left(\sigma_{m_{h}}^{\mathrm{O}} R\right) \\
& \cdot \int_{-\pi}^{\pi} e^{i\left(n^{\prime}-n\right) \phi} d \phi \int_{0}^{h_{\mathrm{C}}} \sin \left(k_{z m_{h}}^{\mathrm{OB}} z\right) \\
& \cdot \sin \left(\frac{m \pi}{h_{\mathrm{C}}} z\right) d z
\end{aligned}
$$

With the help of (132) for the puck field on the LHS of (182b), and employing (191) and (192), the final perpendicular source equation is written as

$$
\begin{aligned}
& { }_{z}^{h} A_{n m N}^{1}(R) a_{n m 0}^{1}+{ }_{z}^{h} A_{n m N}^{2}(R) a_{n m 0}^{2} \\
& =\frac{1}{i \pi h_{\mathrm{C}}} \sin \left(\frac{m \pi z^{\prime}}{h_{\mathrm{C}}}\right) H_{z^{\prime}}^{p t} \Delta \phi^{\prime} e^{-i n \phi^{\prime}}+\frac{2}{i h_{\mathrm{C}}} \\
& \quad \sum_{m_{h}=1}^{M_{\mathrm{O}}^{h_{2}}} K_{n}\left(\sigma_{m_{h}}^{\mathrm{O}} R\right) R_{m_{h}}^{\mathrm{BT}-} A_{n m_{h}}^{h \mathrm{OT}-} I_{s Z m m_{h_{h}}}^{\mathrm{CO}} \\
& m=1,2, \cdots, M_{\mathrm{C}} .
\end{aligned}
$$


The overlap integral in (193) is given by

$$
\begin{aligned}
I_{s Z m m_{h}}^{\mathrm{CO}} & =\int_{0}^{h_{\mathrm{C}}} \sin \left(k_{z m}^{\mathrm{C}} z\right) \bar{Z}_{m_{h}}^{h}(z) d z \\
& =2 i \int_{0}^{h_{\mathrm{C}}} \sin \left(k_{z m}^{\mathrm{C}} z\right) \sin \left(k_{z m_{h}}^{\mathrm{OB}} z\right) d z \\
& =\frac{2 i k_{z m}^{\mathrm{C}}}{\left(k_{z m_{h}}^{\mathrm{OB}}\right)^{2}-\left(k_{z m}^{\mathrm{C}}\right)^{2}}(-1)^{m} \sin \left(k_{z m_{h}}^{\mathrm{OB}} h_{\mathrm{C}}\right) .
\end{aligned}
$$

Orthogonality relationships implicitly contained in (187) and (193) for the puck test eigenfunctions are

$$
\begin{aligned}
\int_{0}^{h_{\mathrm{C}}} & \psi_{h z m}^{\mathrm{C}} \psi_{h z m^{\prime}}^{\mathrm{C}} d z \\
\quad & \int_{0}^{h_{\mathrm{C}}} \sin \left(k_{z m}^{\mathrm{C}} z\right) \sin \left(k_{z m^{\prime}}^{\mathrm{C}} z\right) d z=\frac{h_{\mathrm{C}}}{2} \delta_{m m^{\prime}} \\
\int_{0}^{h_{\mathrm{C}}} \psi_{h \phi m}^{\mathrm{C}} \psi_{h \phi m^{\prime}}^{\mathrm{C}}>0 & \\
\quad & \int_{0}^{h_{\mathrm{C}}} \cos \left(k_{z m}^{\mathrm{C}} z\right) \cos \left(k_{z m^{\prime}}^{\mathrm{C}} z\right) d z \\
& =\frac{h_{\mathrm{C}}}{2 \varepsilon_{m}} \delta_{m m^{\prime}}
\end{aligned}
$$

\section{Complete-System Equations}

The six system equations, four sourceless as seen in (150)-(153), and two with magnetic sources in (187) and (193), may be stated in compact form in the following single matrix formula:

$$
\begin{gathered}
{\left[\begin{array}{cccccc}
0_{a 1} & 0_{a 2} & 1_{e \mathrm{I}} & 0_{h \mathrm{I}} & \sum_{e \mathrm{OT}} & 0_{h \mathrm{OT}} \\
0_{a 1} & 0_{a 2} & 1_{e \mathrm{I}} & 1_{h \mathrm{I}} & \sum_{e \mathrm{OT}} & \sum_{h \mathrm{OT}} \\
\sum_{a 1} & \sum_{a 2} & \sum_{e 1} & 0_{h \mathrm{I}} & 1_{e \mathrm{OT}} & 0_{h \mathrm{OT}} \\
\sum_{a 1} & \sum_{a 2} & \sum_{e 1} & \sum_{h \mathrm{I}} & 1_{e \mathrm{OT}} & 1_{h \mathrm{OT}} \\
1_{a 1} & 1_{a 2} & 0_{e \mathrm{I}} & 0_{h \mathrm{I}} & 0_{e \mathrm{OT}} & \sum_{h \mathrm{OT}} \\
1_{a 2} & 0_{e \mathrm{I}} & 0_{h \mathrm{I}} & \sum_{e \mathrm{OT}} & \sum_{h \mathrm{OT}}
\end{array}\right]\left[\begin{array}{c}
a_{n m 0}^{1} \\
a_{n m 0}^{2} \\
A_{n m}^{e \mathrm{I}} \\
A_{n m}^{h \mathrm{I}} \\
A_{n m}^{e \mathrm{O}} \\
A_{n m}^{h \mathrm{O}}
\end{array}\right]} \\
= \\
\left.\begin{array}{c}
0 \\
\frac{1}{i \pi h_{\mathrm{C}}} \sin \left(\frac{m \pi z^{\prime}}{h{ }_{\mathrm{C}}}\right) H_{z^{\prime}}^{p t} \Delta \phi^{\prime} e^{-i n \phi^{\prime}} \\
\frac{\varepsilon_{m}}{\pi h_{\mathrm{C}}} \cos \left(\frac{m \pi z^{\prime}}{h_{\mathrm{C}}}\right) H_{\phi^{\prime}}^{p t} \Delta \phi^{\prime} e^{-i n \phi^{\prime}}
\end{array}\right] .
\end{gathered}
$$

A representative $n m$ th element for each class of unknowns is shown in the LHS column vector. The matrix entries are as follows: 0 for no contribution of the subscript type of unknown, 1 for a single entry contribution, and $\Sigma$ for a sum of all that particular class of unknowns indicated by the subscript. On the RHS of the equation are the source-forcing terms. It is possible to reduce the size by analytical effort of the system matrix (197) due to the appearance of null and unity entries, and solve a smaller inhomogeneous linear matrix problem, albeit with fewer unknowns determined initially. The remaining unknowns are captured by solving subsidiary matrix equations of generally smaller size than the reduced system matrix.

Setting $H_{z^{\prime}}^{p t} \Delta \phi^{\prime}=1$ and $H_{\phi^{\prime}}^{p t} \Delta \phi=0$ (or the reverse) allows for the solution of the suite of unknowns coefficients in the column vector. When these solutions (or the reverse) are placed in the electromagnetic-field formulas, the dyadic Green's function elements are generated. This may be put down symbolically as

$$
\begin{aligned}
& G_{E, H ; H}^{r, \phi, z ; z}(r, \phi, z)=F_{E, H}^{r, \phi, z}[S(1,0)] \\
& G_{E, H ; H}^{r, \phi, z ; \phi}(r, \phi, z)=F_{E, H}^{r, \phi, z}[S(0,1)] .
\end{aligned}
$$

$S$ represents system (197) and $S(1,0)$ corresponds to the azimuthal magnetic source turned off. $F$ is the field equation operator and produces the correct component (first superscript) for the desired field (electric or magnetic field, indicated by the first subscript).

It is clear that if we had only one term for each summation, then the compressed form in (197) would represent a $6 \times 6$ system, and it would be possible, but extremely tedious, to pull the forcing terms through the determinant solution for each unknown and obtain explicit real-space dyadic Green's function elements. But for our problem here with incommensurate heights (or other geometrical dimensions), the problem is in practice impossible to solve for an explicit dyadic Green's function. One would look naturally to reciprocal space to obtain by analytical means compact explicit dyadic Green's function expressions. However, there is a tremendous advantage in not going to reciprocal space, and this is that the implicit dyadic Green's function can be used to obtain the field behavior directly without any transformations. Furthermore, these Green's functions can be used to obtain the $s$-parameters for the circulator structure too.

Mention is made here that the diagonal nature of the relation between different region amplitudes in the outer zone allows one amplitude (per mode class) to characterize the entire zone (the "T" amplitude was chosen). Once they are determined by (197), the subsidiary diagonal equations (100) and (112), stated explicitly below, may be employed to capture the "B" amplitudes

$$
\left[\begin{array}{c}
A_{n 1}^{e \mathrm{OB}-} \\
A_{n 2}^{e \mathrm{OB}-} \\
\vdots \\
A_{n M_{\mathrm{O}}^{e}}^{e \mathrm{OB}-}
\end{array}\right]=\left[\begin{array}{cccc}
R_{1}^{\mathrm{BT}-} & 0 & \cdots & 0 \\
0 & R_{2}^{\mathrm{BT}-} & \cdots & 0 \\
\vdots & \vdots & \ddots & \vdots \\
0 & 0 & \cdots & R_{M_{\mathrm{O}}^{e}}^{\mathrm{BT}-}
\end{array}\right]\left[\begin{array}{c}
A_{n 1}^{e \mathrm{OT}-} \\
A_{n 2}^{e \mathrm{~T}-} \\
\vdots \\
A_{n M_{\mathrm{O}}^{e}}^{e \mathrm{TT}-}
\end{array}\right]
$$

$$
\left[\begin{array}{c}
A_{n 1}^{h \mathrm{OB}-} \\
A_{n 2}^{h \mathrm{OB}-} \\
\vdots \\
A_{n M_{\mathrm{O}}^{h}}^{h \mathrm{OB}-}
\end{array}\right]=\left[\begin{array}{cccc}
R_{1}^{\mathrm{BT}-} & 0 & \cdots & 0 \\
0 & R_{2}^{\mathrm{BT}-} & \cdots & 0 \\
\vdots & \vdots & \ddots & \vdots \\
0 & 0 & \cdots & R_{M_{\mathrm{O}}^{h_{2}}}^{\mathrm{BT}}
\end{array}\right]\left[\begin{array}{c}
A_{n \mathrm{OT}-}^{h \mathrm{OT}-} \\
A_{n 2}^{h 2} \\
\vdots \\
A_{n M_{\mathrm{O}}^{h}}^{h \mathrm{OT}-}
\end{array}\right] .
$$

\section{CONClusion}

Source-constraint equations have been combined with the mode-matching technique to obtain in direct space implicit dyadic Green's function elements for a very general canonical circulator geometry. The approach allows the inclusion of 
layered surrounding material beyond the radius of the ferrite puck, as well as a covering material above the puck, enabling a more realistic or complete description of the circulator structure. New self-adjoint operators are found consistent with the surrounding material having dielectric, magnetic, or simultaneous character. Inclusion of substrate and superstrate effects are possible with this treatment. Assessment of vertical field fringing is a natural consequence of this analysis approach. Obtaining the dyadic Green's function as described in the paper is particularly useful because field information and $s$-paramaters may be found in real space, completely avoiding typical inverse transformations.

Included in this approach are the inhomogeneous properties of the circulator puck due to chosen radial variation of the ferrite material parameters, nonuniform applied magnetic dcbiasing field, or finite puck geometric effects on the bias field. All of these inhomogeneous properties in the puck region are naturally incorporated into the dyadic Green's function. Numerical evaluation should be efficient and only limited by the well understood features of the mode-matching technique.

The geometry considered corresponds to that found when one of two self-biasing configurations are employed using hexagonal ferrite films [15]-[17]. The puck itself is a hexagonal material with the exterior zone a layered combination of materials or the puck is spinel or garnet material and the exterior material a hexagonal material (it may be anisotropic, a situation not treated here). In the first structure, the electricwall condition above the puck is maintained by microstrip metal. For the second structure, it is maintained by a combination of the microstrip metal and low-coercivity "keeper" plate (or cover) permalloy. Hexagonal materials include $\mathrm{Ba}$, $\mathrm{Pb}$, or $\mathrm{Sr}$, iron-oxide compounds. The garnets are the usual $\mathrm{Y}$, iron-oxide compounds, and the spinels are the $\mathrm{Ni}, \mathrm{Li}$, or $\mathrm{MgMn}$, iron-oxide compounds.

\section{REFERENCES}

[1] C. M. Krowne and R. E. Neidert, "Theory and numerical calculations for radially inhomogeneous circular ferrite circulators," IEEE Trans. Microwave Theory Tech., vol. 44, pp. 419-431, Mar. 1996.

[2] C. M. Krowne, "3D dyadic Green's function for radially inhomogeneous circular ferrite circulator," in IEEE Microwave Theory Tech. Symp. Dig., San Francisco, CA, June 18, 1996, pp. 121-124.

[3] D. Maystre, P. Vincent, and J. C. Mage, "Theoretical and experimental study of the resonant frequency of a cylindrical dielectric resonator," IEEE Trans. Microwave Theory Tech., vol. 31, pp. 844-848, Oct. 1983.

[4] W. K. Hui and I. Wolff, "Dielectric ring-gap resonator for application in MMIC's," IEEE Trans. Microwave Theory Tech., vol. 39, pp. 2061-2068, Nov. 1991.

[5] _ "A multicomposite, multilayered cylindrical dielectric resonator for application in MMIC's," IEEE Trans. Microwave Theory Tech., vol. 42, pp. 415-423, Mar. 1994.

[6] K. A. Michalski, "Rigorous analysis methods," in Dielectric Resonators, D. Kajfez and P. Guillon, Eds. Norwood, MA: Artech House, 1986, pp. $185-258$

[7] P. M. Morse and H. Feshbach, Part I: Methods of Theoretical Physics. New York: McGraw-Hill, 1953.
[8] B. Friedman, Principles and Techniques of Applied Mathematics. New York: Wiley, 1956

[9] R. Courant, "Partial differential equations," in Part II: Methods of Mathematical Physics, by R. Courant and D. Hilbert. New York: Interscience, 1962

[10] C. M. Krowne, "Theory of the recursive dyadic Green's function for inhomogeneous ferrite canonically shaped microstrip circulators," in Advances in Imaging and Electron Physics, Peter W. Hawkes, Ed. New York: Academic, 1996, pp. 77-321.

[11] I. S. Gradshteyn and I. M. Ryzhik, Table of Integrals, Series, and Products. New York: Academic, 1980.

[12] J. J. H. Wang, Generalized Moment Methods in Electromagnetics. New York: Wiley, 1996.

[13] T. S. Chu, T. Itoh, and Y.-C. Shih, "Comparative study of modematching formulations for microstrip discontinuity problems," IEEE Trans. Microwave Theory Tech., vol. MTT-33, pp. 1018-1023, Oct. 1985.

[14] J. R. Mautz, "On the electromagnetic field in a cavity fed by a tangential electric field in an aperture in its wall," IEEE Trans. Microwave Theory Tech., vol. 43, pp. 620-626, Mar. 1995

[15] C. A. Carosella, D. B. Chrisey, P. Lubitz, J. S. Horwitz, P. Dorsey, R. Seed, and C. Vittoria, "Pulsed laser deposition of epitaxial $\mathrm{BaFe}_{12} \mathrm{O}_{19}$ thin films," J. Appl. Phys., vol. 71, no. 10, pp. 5107-5110, May 15, 1992

[16] R. Karim, S. D. Ball, J. R. Truedson, and C. E. Patton, "Frequency dependence of the ferromagnetic resonance linewidth and effective linewidth in maganese substituted single crystal barium ferrite," J. Appl. Phys., vol. 73, no. 9, pp. 4512-4515, May 1993.

[17] A. Morisako, H. Nakanishi, M. Matsumoto, and M. Naoe, "Lowtemperature deposition of hexagonal ferrite films by sputtering," J. Appl. Phys., vol. 75, no. 10, pp. 5969-5971, May 1994.

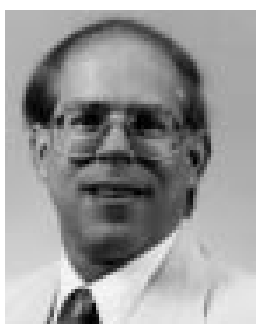

Clifford M. Krowne (S'73-M'74-SM'83) received the B.S. degree from the University of California at Berkeley and Davis, in 1970, the M.S. and Ph.D degrees from the University of California at Los Angeles, in 1972 and 1975, respectively.

He worked in the Microelectronics Division, Lockheed Missiles and Space Company, Sunnyvale, CA, as a Member of the Solid-State Technical Staff, Watkins-Johnson Company, Palo Alto, CA, and as a Faculty Member in the Department of Electrical Engineering, North Carolina State University, Raleigh. Since 1982, he has been with the Microwave Technology Branch, Electronics Science and Technology Division, Naval Research Laboratory, Washington, DC, studying microwave and millimeter-wave properties of active and passive solid-state devices. He was also an Adjunct Professor of electrical engineering at the University of Maryland at College Park. He has authored or co-authored 147 conference papers, books, and journal papers, and holds several patents on solid-state electronics, microwave circuits, electromagnetics, engineering education, and applied physics. He has also authored the major portions of two books for Academic Press in the Advances in Imaging and Electron Physics Series (vol. 92, 1995; vol. 98, 1996).

Dr. Krowne is a member of Phi Kappa Phi and Tau Beta Pi, and is a Fellow of the Washington Academy of Sciences. He has served on the technical program conference committees of the IEEE Antennas and Propagation Society $(1983,1984)$ and the IEEE Microwave Theory and Techniques Society (1982-1996), has chaired sessions in the electromagnetic theory, microstrip antenna, and solid-state devices/circuits, superconductor, and monolithic circuits areas, and has organized two MTT-S workshops on 2-D/3-D full-wave simulation (1992), and self-consistent particle transport/full-wave dynamic field simulation (1993). He was a member of the 1987 IEEE Microwave Theory and Techniques Symposium Steering Committee. He is listed in Who's Who in Frontiers of Science and Technology and Who's Who in Electromagnetics. 